Pacific

Journal of

Mathematics

\title{
MAPS FROM THE ENVELOPING ALGEBRA OF THE POSITIVE WITT ALGEBRA TO REGULAR ALGEBRAS
}

Susan J. Sierra ANd Chelsea Walton 


\title{
MAPS FROM THE ENVELOPING ALGEBRA OF THE POSITIVE WITT ALGEBRA TO REGULAR ALGEBRAS
}

\author{
SusAn J. SierRa AND Chelsea WALton
}

\begin{abstract}
We construct homomorphisms from the universal enveloping algebra of the positive (part of the) Witt algebra to several different Artin-Schelter regular algebras, and determine their kernels and images. As a result, we produce elementary proofs that the universal enveloping algebras of the Virasoro algebra, the Witt algebra, and the positive Witt algebra are neither left nor right noetherian.
\end{abstract}

\section{Introduction}

Let $\mathbb{k}$ be a field of characteristic 0 . All vector spaces, algebras, and tensor products are over $\mathbb{k}$, unless stated otherwise. In this work, we construct and study homomorphisms from the universal enveloping algebra of the positive part of the Witt algebra to Artin-Schelter (AS-)regular algebras. The latter serve as homological analogues of commutative polynomial rings in the field of noncommutative algebraic geometry.

To begin, consider the Lie algebras below.

Definition 0.1 $\left(V, W, W_{+}\right)$. We define the following Lie algebras:

(a) The Virasoro algebra is defined to be the Lie algebra $V$ with basis $\left\{e_{n}\right\}_{n \in \mathbb{Z}} \cup\{c\}$ and Lie bracket $\left[e_{n}, c\right]=0,\left[e_{n}, e_{m}\right]=(m-n) e_{n+m}+\frac{1}{12} c\left(m^{3}-m\right) \delta_{n+m, 0}$.

(b) The Witt (or centerless Virasoro) algebra is defined to be the Lie algebra $W$ with basis $\left\{e_{n}\right\}_{n \in \mathbb{Z}}$ and Lie bracket $\left[e_{n}, e_{m}\right]=(m-n) e_{n+m}$.

(c) The positive (part of the) Witt algebra is defined to be the Lie subalgebra $W_{+}$ of $W$ generated by $\left\{e_{n}\right\}_{n \geq 1}$.

For any Lie algebra $\mathfrak{g}$, we denote its universal enveloping algebra by $U(\mathfrak{g})$.

Further, consider the following algebras.

MSC2010: 14A22, 16S30, 16S38, 17B68.

Keywords: Artin-Schelter regular, Jordan plane, non-noetherian, universal enveloping algebra, Witt algebra. 
Notation $0.2(S, R)$. Let $S$ be the algebra generated by $u, v, w$, subject to the relations

$$
u v-v u-v^{2}=u w-w u-v w=v w-w v=0 .
$$

Let $R$ be the Jordan plane generated by $u, v$, subject to the relation $u v-v u-v^{2}=0$.

It is well known that $R$ is an AS-regular algebra of global dimension 2. Moreover, we see by Lemma 1.3 that $S$ is also AS-regular, of global dimension 3 .

This work focuses on maps that we construct from the enveloping algebra $U\left(W_{+}\right)$ to both $R$ and $S$, given as follows:

Definition $0.3\left(\phi, \lambda_{a}\right)$. Let $\phi: U\left(W_{+}\right) \rightarrow S$ be the algebra homomorphism induced by defining

$$
\phi\left(e_{n}\right)=(u-(n-1) w) v^{n-1} .
$$

For $a \in \mathbb{k}$, let $\lambda_{a}: U\left(W_{+}\right) \rightarrow R$ be the algebra homomorphism induced by defining

$$
\lambda_{a}\left(e_{n}\right)=(u-(n-1) a v) v^{n-1} .
$$

That $\phi$ and $\lambda_{a}$ are well defined is Lemma 1.5.

Our main result is that we understand fully the kernels and images of the maps above, as presented below.

Theorem 0.6. We have the following statements about the kernels and images of the maps $\phi$ and $\lambda_{a}$.

(a) [Propositions 2.5, 2.8] ker $\lambda_{a}$ is equal to the ideal $\left(e_{1} e_{3}-e_{2}^{2}-e_{4}\right)$ if $a=0,1$; or is an ideal generated by one element of degree 5 and two elements of degree 6 (listed in Proposition 2.8) if $a \neq 0,1$.

(b) [Proposition 2.1] $\lambda_{a}\left(U\left(W_{+}\right)\right)$is equal to $\mathbb{k}+u R$ if $a=0$; is equal to $\mathbb{k}+R u$ if $a=1$; or contains $R_{\geq 4}$ if $a \neq 0,1$. For all $a$, the image of $\lambda_{a}$ is noetherian.

(c) [Theorem 5.1] ker $\phi$ is equal to $\left(e_{1} e_{5}-4 e_{2} e_{4}+3 e_{3}^{2}+2 e_{6}\right)$.

The image of $\phi$ will be discussed later in the introduction, after Theorem 0.10. The result above has a surprising application. In [Sierra and Walton 2014, Theorem 0.5 and Corollary 0.6], we established that $U\left(W_{+}\right), U(W), U(V)$ are neither left nor right noetherian through relatively indirect means, using the techniques of [Sierra 2011]. In particular, we were not able to give an example of a non-finitelygenerated right or left ideal in any of these enveloping algebras. However, in the course of proving Theorem 0.6, we produce an elementary and constructive proof of [Sierra and Walton 2014, Theorem 0.5 and Corollary 0.6]. Namely, we obtain:

Theorem 0.7 (Proposition 2.5, Theorem 3.3). The ideal

$$
\operatorname{ker} \lambda_{0}=\operatorname{ker} \lambda_{1}=\left(e_{1} e_{3}-e_{2}^{2}-e_{4}\right)
$$

is not finitely generated as either a left or a right ideal of $U\left(W_{+}\right)$. 
We prove this theorem by noting that $\lambda_{0}$ factors through $\phi$, and by studying $B:=\phi\left(U\left(W_{+}\right)\right)$. A key step is to compute $I:=\phi\left(\operatorname{ker} \lambda_{0}\right)$, and to show that $I$ is not finitely generated as a left or right ideal of $B$.

Note that the map (0.5) can be extended to $W$ to define a map, which we denote by

$$
\hat{\lambda}_{a}: U(W) \rightarrow R\left[v^{-1}\right] .
$$

Theorem 0.8 ((3.10), Theorem 3.12). The ideal ker $\hat{\lambda}_{0}=\operatorname{ker} \hat{\lambda}_{1}$ is not finitely generated as either a left or right ideal of $U(W)$.

We remark that $R\left[v^{-1}\right]$ is isomorphic to the ring $\mathbb{k}\left[x, x^{-1}, \partial\right]$, which is a familiar localization of the Weyl algebra. To see this, set $v=x$ and $u=x^{2} \partial$, so $\partial x=x \partial+1$. Then $u v-v u=x^{2}=v^{2}$. We obtain

$$
\hat{\lambda}_{1}\left(e_{n}\right)=v^{n-1} u=x^{n+1} \partial .
$$

Thus, $\hat{\lambda}_{1}$ is a well-known homomorphism.

We now compare Theorem 0.7 with our earlier proof (in [Sierra and Walton 2014]) that $U\left(W_{+}\right)$is not left or right noetherian. The earlier proof used a ring homomorphism $\rho$ with a more complicated definition:

Notation 0.9 $(X, f, \tau, \rho)$. Take $\mathbb{P}^{3}:=\mathbb{P}_{\mathbb{k}}^{3}$ with coordinates $w, x, y, z$. Let $X=$ $V\left(x z-y^{2}\right) \subseteq \mathbb{P}^{3}$ be the projective cone over a smooth conic in $\mathbb{P}^{2}$.

Define an automorphism $\tau$ of $X$ by

$$
\tau([w: x: y: z])=[w-2 x+2 z: z:-y-2 z: x+4 y+4 z] .
$$

Denote the pullback of $\tau$ on $\mathbb{k}(X)$ by $\tau^{*}$, so that $g^{\tau}:=\tau^{*} g=g \circ \tau$ for $g \in \mathbb{k}(X)$. Form the ring $\mathbb{k}(X)\left[t ; \tau^{*}\right]$ with multiplication $t g=g^{\tau} t$ for all $g \in \mathbb{k}(X)$. Let

$$
f:=\frac{w+12 x+22 y+8 z}{12 x+6 y},
$$

considered as a rational function in $\mathbb{k}(X)$. Now let $\rho: U\left(W_{+}\right) \rightarrow \mathbb{k}(X)\left[t ; \tau^{*}\right]$ be the algebra homomorphism induced by setting $\rho\left(e_{1}\right)=t$ and $\rho\left(e_{2}\right)=f t^{2}$.

That $\rho$ is well defined is [Sierra and Walton 2014, Proposition 1.5]. The method in that paper made a number of reductions to show that $\rho\left(U\left(W_{+}\right)\right)$is not left or right noetherian. That proof can now be streamlined via the next result.

Theorem 0.10 (Theorem 4.1). We have that $\operatorname{ker} \rho=\operatorname{ker} \phi=\bigcap_{a \in \mathbb{k}} \operatorname{ker} \lambda_{a}$.

Since we show that $\phi\left(U\left(W_{+}\right)\right)$is not left or right noetherian in the course of proving Theorem 0.7, we have by Theorems $0.6(\mathrm{c})$ and 0.10 that $\rho\left(U\left(W_{+}\right)\right) \cong$ $\phi\left(U\left(W_{+}\right)\right) \cong U\left(W_{+}\right) /\left(e_{1} e_{5}-4 e_{2} e_{4}+3 e_{3}^{2}+2 e_{6}\right)$ is neither left nor right noetherian.

We end by discussing an open question that was brought to our attention by Lance Small. 
Question 0.11. Does $U\left(W_{+}\right)$satisfy the ascending chain condition on two-sided ideals?

Our result here is only partial:

Proposition 0.12 (Proposition 6.6). The ring $B:=\phi\left(U\left(W_{+}\right)\right)$satisfies the ascending chain condition on two-sided ideals.

Of course, this yields no direct information on the question for $U\left(W_{+}\right)$.

We have the following conventions throughout the paper. We take $\mathbb{N}=\mathbb{Z}_{\geq 0}$ to be the set of nonnegative integers. If $r$ is an element of a ring $A$, then $(r)$ denotes the two-sided ideal $A r A$ generated by $r$. If $A=\bigoplus_{n \in \mathbb{Z}} A_{n}$ is a graded k-algebra (or graded module), then we define the Hilbert series

$$
\text { hilb } A=\sum_{n \in \mathbb{Z}} \operatorname{dim}_{\mathbb{k}} A_{n} t^{n} .
$$

This article is organized as follows. We present preliminary results in Section 1, including an alternative way of multiplying elements in $S$ and in $R$ (Lemma 1.3); this method will be employed throughout, sometimes without mention. In Section 2, we discuss the maps $\lambda_{a}$ and prove parts (a) and (b) of Theorem 0.6. In Section 3 we use the map $\lambda_{0}$ to establish Theorem 0.7; we also prove Theorem 0.8.

Before proceeding to study the map $\phi$, we present its connection with the map $\rho$, the key homomorphism of [Sierra and Walton 2014]. Namely, in Section 4, we establish Theorem 0.10. Then in Section 5, we verify part (c) of Theorem 0.6. Our last result, Proposition 0.12, is presented in Section 6. Proofs of computational claims via Maple and Macaulay2 routines and a known result in ring theory to which we could not find a reference are provided in the Appendix.

\section{Preliminaries}

The main focus of this paper is the universal enveloping algebra of the positive Witt algebra, $W_{+}$. To begin, we recall some basic facts about the algebra $U\left(W_{+}\right)$.

Lemma 1.1. Recall Definition 0.1(c).

(a) We have the isomorphism

$$
U\left(W_{+}\right) \cong \frac{\mathbb{k}\left\langle e_{1}, e_{2}\right\rangle}{\left(\begin{array}{c}
{\left[e_{1},\left[e_{1},\left[e_{1}, e_{2}\right]\right]\right]+6\left[e_{2},\left[e_{2}, e_{1}\right]\right],} \\
{\left[e_{1},\left[e_{1},\left[e_{1},\left[e_{1},\left[e_{1}, e_{2}\right]\right]\right]\right]\right]+40\left[e_{2},\left[e_{2},\left[e_{2}, e_{1}\right]\right]\right]}
\end{array}\right)} .
$$

(b) The set $\left\{e_{i_{1}}, e_{i_{2}}, \ldots, e_{i_{k}} \mid k \in \mathbb{N}\right.$ and $\left.1 \leq i_{1} \leq i_{2} \leq \cdots \leq i_{k} \in \mathbb{N}\right\}$ forms $a$ $\mathbb{k}$-vector space basis of $U\left(W_{+}\right)$.

Proof. Part (a) is [Sierra and Walton 2014, Lemma 1.1], and part (b) is clear from the definition of $U\left(W_{+}\right)$. 
Next, let us present some notation that we will use for the rest of the paper. We will work with the algebras $R$ and $S$ defined in Notation 0.2 ; note that we can view $R$ as a subalgebra of $S$. In addition:

Notation 1.2 $(Q)$. Take $Q$ to be the subalgebra of $S$ generated by $u, v$, and $v w$.

In our first result, we provide an easy way to multiply elements in $S$. Recall from [Zhang 1996] that a Zhang twist of a graded algebra $L$, by an automorphism $\mu$ of $L$, is the algebra $L^{\mu}$, where $L^{\mu}=L$ as graded vector spaces and $L^{\mu}$ has multiplication $\ell * \ell^{\prime}=\ell\left(\ell^{\prime}\right)^{\mu^{i}}$ for $\ell \in L_{i}$ and $\ell^{\prime} \in L$.

Moreover, recall that an Artin-Schelter (AS-)regular algebra is a connected graded algebra $A$ of finite global dimension, of finite injective dimension $d$ with $\operatorname{Ext}_{A}^{i}\left({ }_{A} \mathbb{k},{ }_{A} A\right) \cong \operatorname{Ext}_{A}^{i}\left(\mathbb{k}_{A}, A_{A}\right) \cong \delta_{i, d} \mathbb{k}$ (that is, $A$ is $A S$-Gorenstein), and has finite Gelfand-Kirillov dimension. These algebras are important in noncommutative ring theory because they are noncommutative analogues of polynomial rings and share many of their good properties.

Lemma $1.3(\mu, v)$. Let $\mu \in \operatorname{Aut}(\mathbb{k}[x, y, z])$ be defined by

$$
\mu(x)=x-y, \quad \mu(y)=y, \quad \mu(z)=z .
$$

Then $S$ is isomorphic to the Zhang twist $\mathbb{k}[x, y, z]^{\mu}$. Further, $\mu$ restricts to an automorphism of $\mathbb{k}[x, y, y z]$, which we also denote by $\mu$, and to an automorphism $v$ of $\mathbb{k}[x, y]$. We have that $R \cong \mathbb{k}[x, y]^{v}$ and $Q \cong \mathbb{k}[x, y, y z]^{\mu}$ as graded $\mathbb{k}$-algebras. As a consequence, $S, R$, and $Q$ are $A S$-regular algebras.

Proof. To see that $S \cong \mathbb{k}[x, y, z]^{\mu}$, we emphasize that

- the variables $u, v, w$ of $S$ have noncommutative multiplication,

- the variables $x, y, z$ of $\mathbb{k}[x, y, z]$ have commutative multiplication, and

- the symbol $*$ denotes the noncommutative multiplication on $\mathbb{k}[x, y, z]^{\mu}$ defined by $\ell * \ell^{\prime}=\ell\left(\ell^{\prime}\right)^{\mu^{i}}$ for $\ell \in \mathbb{k}[x, y, z]_{i}$ and $\ell^{\prime} \in \mathbb{k}[x, y, z]$.

Now,

$$
\begin{aligned}
& y * x=y x^{\mu}=y(x-y)=(x-y) y=x y-y^{2}=x y^{\mu}-y y^{\mu}=x * y-y * y, \\
& z * x=z x^{\mu}=z(x-y)=(x-y) z=x z-y z=x z^{\mu}-y z^{\mu}=x * z-y * z, \\
& z * y=z y^{\mu}=z y \quad=y z \quad=y z^{\mu} \quad=y * z .
\end{aligned}
$$

Thus, if we identify $u, v, w$ with $x, y, z$, respectively, then the relations of $S$ hold in $\mathbb{k}[x, y, z]^{\mu}$, and $S \cong \mathbb{k}[x, y, z]^{\mu}$ as graded $\mathbb{k}$-algebras.

That $\mu$ restricts to automorphisms of $\mathbb{k}[x, y]$ and $\mathbb{k}[x, y, y z]$ is immediate, and the other isomorphisms hold by a similar argument. Moreover, the last statement follows as commutative polynomial rings are AS-regular and this property is preserved under Zhang twisting by [Zhang 1996, Theorem 1.3(i)]. 
Now we verify that the algebra homomorphisms $\lambda_{a}$ and $\phi$ from Definition 0.3 are well defined.

Lemma 1.5. The maps $\phi$ and $\lambda_{a}$ of Definition 0.3 are well-defined homomorphisms of graded $\mathbb{k}$-algebras.

Proof. We check that $\phi$ respects the Witt relations given in Definition 0.1(b), by using Lemma 1.3 and (1.4):

$$
\begin{aligned}
& \phi\left(e_{n} e_{m}-e_{m} e_{n}\right) \\
& =(u-(n-1) w) v^{n-1}(u-(m-1) w) v^{m-1}-(u-(m-1) w) v^{m-1}(u-(n-1) w) v^{n-1} \\
& =(x-(n-1) z)(x-(m-1) z)^{\mu^{n}} y^{n+m-2}-(x-(m-1) z)(x-(n-1) z)^{\mu^{m}} y^{n+m-2} \\
& =((x-(n-1) z)(x-n y-(m-1) z)-(x-(m-1) z)(x-m y-(n-1) z)) y^{n+m-2} \\
& =(m-n) x y^{n+m-1}+(n(n-1)-m(m-1)) y^{n+m-1} z \\
& =(m-n)(x-(n+m-1) z) y^{n+m-1} \\
& =(m-n)(u-(n+m-1) w) v^{n+m-1} \\
& =(m-n) \phi\left(e_{n+m}\right) .
\end{aligned}
$$

So, the claim holds for $\phi$.

Similarly, we verify that $\lambda_{a}$ respects the Witt relations:

$$
\begin{aligned}
\lambda_{a}\left(e_{n} e_{m}-e_{m} e_{n}\right)= & (u-(n-1) a v) v^{n-1}(u-(m-1) a v) v^{m-1} \\
& -(u-(m-1) a v) v^{m-1}(u-(n-1) a v) v^{n-1} \\
= & ((x-(n-1) a y)(x-n y-(m-1) a y) \\
& \quad-(x-(m-1) a y)(x-m y-(n-1) a y)) y^{n+m-2} \\
= & (m-n)(x-a(n+m-1) y) y^{n+m-1} \\
= & (m-n)(u-a(n+m-1) v) v^{n+m-1} \\
= & (m-n) \lambda_{a}\left(e_{n+m}\right) .
\end{aligned}
$$

Thus, the claim holds for $\lambda_{a}$.

Next, we define the key algebras $A(a)$ and $B$ that we will use throughout.

Notation 1.6 $(A(a), B)$. Take $a \in \mathbb{k}$ and let $A(a)$ denote the subalgebra $\lambda_{a}\left(U\left(W_{+}\right)\right)$ of $R$. Further, let $B$ denote the subalgebra $\phi\left(U\left(W_{+}\right)\right)$of $S$.

We point out a useful observation.

Lemma 1.7. We have that $B \subseteq Q$.

Proof. We get that $\phi\left(e_{1}\right)=u$ and $\phi\left(e_{2}\right)=(u-w) v=u v-v w$ are in $Q$. By Lemma 1.1(a), $B$ is generated by these elements, so we are done. 


\section{The kernel and image of the maps $\lambda_{a}$}

The goal of this section is to analyze the maps $\lambda_{a}$ from Definition 0.3, which are well defined by Lemma 1.5. In particular, we verify Theorem 0.6(a,b).

To proceed, recall Notations 0.2 and 1.6. We first compute the factor rings $A(a)$, proving Theorem 0.6(b).

Proposition 2.1. We have that $A(0)=\mathbb{k}+u R$ (a right idealizer in $R$ ), that $A(1)=$ $\mathbb{k}+R u$ (a left idealizer in $R$ ), and that $A(a)_{\geq 4}=R_{\geq 4}$ if $a \neq 0,1$. For all $a$, the ring $A(a)$ is noetherian.

Proof. Recall from Lemma 1.1(a) that $U\left(W_{+}\right)$is generated by $e_{1}$ and $e_{2}$. We have that $\lambda_{0}\left(e_{1}\right)=u$ and $\lambda_{0}\left(e_{2}\right)=u v$. These elements generate $\mathbb{k}+u R$. Moreover, $\lambda_{1}\left(e_{1}\right)=u$ and $\lambda_{1}\left(e_{2}\right)=(u-v) v=v u$, and these elements generate $\mathbb{k}+R u$. That the rings $A(0)$ and $A(1)$ are noetherian follows from [Stafford and Zhang 1994, Lemma 2.2(iii) and Theorem 2.3(i.a)].

When $a \neq 0,1$, we must show that $R_{\geq 4} \subseteq A(a)$. Since $u R_{n}+R_{n} u=R_{n+1}$ for $n \geq 1$ and since $\operatorname{dim}_{\mathbb{k}} R_{4}=5$, the proof boils down to showing that the set of elements

$$
\lambda_{a}\left(e_{1}^{4}\right), \quad \lambda_{a}\left(e_{1}^{2} e_{2}\right), \quad \lambda_{a}\left(e_{1} e_{2} e_{1}\right), \quad \lambda_{a}\left(e_{2} e_{1}^{2}\right), \quad \lambda_{a}\left(e_{2}^{2}\right)
$$

is $\mathbb{k}$-linearly independent for $a \neq 0,1$. Using Lemma 1.3 and (1.4), consider the following calculations:

$$
\begin{aligned}
& \lambda_{a}\left(e_{1}^{4}\right)=u^{4}=x x^{\mu} x^{\mu^{2}} x^{\mu^{3}}=x(x-y)(x-2 y)(x-3 y)=: r_{1}, \\
& \lambda_{a}\left(e_{1}^{2} e_{2}\right)=u^{2}(u-a v) v=x x^{\mu}(x-a y)^{\mu^{2}} y^{\mu^{3}}=x(x-y)(x-(2+a) y) y=: r_{2}, \\
& \lambda_{a}\left(e_{1} e_{2} e_{1}\right)=u(u-a v) v u=x(x-a y)^{\mu} y^{\mu^{2}} x^{\mu^{3}}=x(x-(1+a) y) y(x-3 y)=: r_{3} \text {, } \\
& \lambda_{a}\left(e_{2} e_{1}^{2}\right)=(u-a v) v u^{2}=(x-a y) y^{\mu} x^{\mu^{2}} x^{\mu^{3}}=(x-a y) y(x-2 y)(x-3 y)=: r_{4}, \\
& \lambda_{a}\left(e_{2}^{2}\right)=(u-a v) v(u-a v) v=(x-a y) y^{\mu}(x-a y)^{\mu^{2}} y^{\mu^{3}} \\
& =(x-a y) y(x-(2+a) y) y=: r_{5} .
\end{aligned}
$$

By direct computation, we see that $r_{1}, \ldots, r_{5}$ are linearly independent if $a \neq 0,1$.

Further, since $A(a)$ and $R$ are equal in large degree and $R$ is noetherian, $A(a)$ is noetherian by [Stafford 1985, Lemma 1.4].

Next we compute the kernels of the maps $\lambda_{a}$ and establish Theorem 0.6(a). We will use the following notation:

Notation $2.2\left(\pi, \pi_{a}, \pi_{B}\right)$. Let $\mathbb{k}\left\langle t_{1}, t_{2}\right\rangle$ be the free algebra, which we grade by setting $\operatorname{deg} t_{i}=i$. We set the notation below: 
- $\pi: \mathbb{k}\left\langle t_{1}, t_{2}\right\rangle \rightarrow U\left(W_{+}\right)$is the algebra map given by $\pi\left(t_{1}\right)=e_{1}$ and $\pi\left(t_{2}\right)=e_{2}$.

- $\pi_{a}: \mathbb{k}\left\langle t_{1}, t_{2}\right\rangle \rightarrow R$ is the algebra map given by $\pi_{a}\left(t_{1}\right)=\lambda_{a}\left(e_{1}\right)=u$ and $\pi_{a}\left(t_{2}\right)=\lambda_{a}\left(e_{2}\right)=(u-a v) v$, for $a \in \mathbb{k}$. The image of $\pi_{a}$ is $A(a)$. Note that $\pi_{a}=\lambda_{a} \circ \pi$.

- $\pi_{B}: \mathbb{k}\left\langle t_{1}, t_{2}\right\rangle \rightarrow S$ is the algebra map given by $\pi_{B}\left(t_{1}\right)=\phi\left(e_{1}\right)=u$ and $\pi_{B}\left(t_{2}\right)=\phi\left(e_{2}\right)=u v-v w$. The image of $\pi_{B}$ is $B$. Note that $\pi_{B}=\phi \circ \pi$.

In the next result, we compute a presentation of the algebra $A(0)$.

Lemma 2.3. The kernel of $\pi_{0}$ is generated by

$$
\begin{aligned}
q & :=t_{1}^{2} t_{2}-t_{2} t_{1}^{2}-2 t_{2}^{2}, \\
q^{\prime} & :=t_{1}^{3} t_{2}-3 t_{1}^{2} t_{2} t_{1}+3 t_{1} t_{2} t_{1}^{2}-t_{2} t_{1}^{3}+6 t_{2}^{2} t_{1}-12 t_{2} t_{1} t_{2}+6 t_{1} t_{2}^{2}
\end{aligned}
$$

as a two-sided ideal.

Proof. Let $A=A(0)$, and consider the exact sequence of right $A$-modules

$$
0 \longrightarrow K \longrightarrow A[-1] \oplus A[-2] \stackrel{(u, u v)}{\longrightarrow} A \longrightarrow \mathbb{k} \longrightarrow 0 .
$$

Claim. As a right $A$-module, $K$ is generated by

$$
\left(u^{2} v,-u(u+2 v)\right) \text { and }\left(u^{2} v^{2},-u(u+2 v) v\right) .
$$

Assume the claim. It is well known that one may deduce generators and relations of a connected graded $\mathbb{k}$-algebra from the first few terms in a minimal resolution of the trivial module $\mathbb{k}$. The precise method is given in Proposition A.1 in the Appendix. Using the notation of that result, take

$$
\begin{array}{ll}
b_{1}^{1}=u^{2} v, & b_{2}^{1}=-u(u+2 v), \\
b_{1}^{2}=u^{2} v^{2}, & b_{2}^{2}=-u(u+2 v) v .
\end{array}
$$

Moreover, take

$$
\begin{array}{ll}
\tilde{b}_{1}^{1}=t_{1} t_{2}, & \tilde{b}_{2}^{1}=-t_{1}^{2}-2 t_{2}, \\
\tilde{b}_{1}^{2}=t_{1}^{2} t_{2}-t_{1} t_{2} t_{1}, & \tilde{b}_{2}^{2}=2 t_{2} t_{1}-3 t_{1} t_{2} .
\end{array}
$$

Note that $\pi_{0}\left(\tilde{b}_{j}^{i}\right)=b_{j}^{i}$ for $i, j=1,2$. Now we obtain by Proposition A.1 that

$$
\begin{aligned}
& q_{1}:=t_{1}\left(\tilde{b}_{1}^{1}\right)+t_{2}\left(\tilde{b}_{2}^{1}\right)=t_{1}^{2} t_{2}-t_{2} t_{1}^{2}-2 t_{2}^{2}, \\
& q_{2}:=t_{1}\left(\tilde{b}_{1}^{2}\right)+t_{2}\left(\tilde{b}_{2}^{2}\right)=t_{1}^{3} t_{2}-t_{1}^{2} t_{2} t_{1}+2 t_{2}^{2} t_{1}-3 t_{2} t_{1} t_{2}
\end{aligned}
$$

generate $\operatorname{ker} \pi_{0}$. Observe that $q=q_{1}$ and that

$q^{\prime}-4 q_{2}=-3 t_{1}^{3} t_{2}+t_{1}^{2} t_{2} t_{1}+3 t_{1} t_{2} t_{1}^{2}-t_{2} t_{1}^{3}-2 t_{2}^{2} t_{1}+6 t_{1} t_{2}^{2}=-3 t_{1} q+q t_{1} \in(q)$.

Thus, ker $\pi_{0}$ is generated by $q$ and $q^{\prime}$, as desired. 
So it remains to prove the claim.

Proof of claim. Note that there is an isomorphism of graded right $A$-modules $\beta: u A \cap u v A \rightarrow K$ given by $\beta(r)=\left(u^{-1} r,-(u v)^{-1} r\right)$.

Take $M:=A \cap v A$. Since $A=\mathbb{k}+u R$, it is easy to show that $M=u R \cap v u R$, and in particular, that $M$ is a right $R$-module. Since $(u R+v u R)_{\geq 2}=R_{\geq 2}$, we get that

$$
\operatorname{dim}_{\mathbb{k}} M_{n}=\operatorname{dim}_{\mathbb{k}} R_{n-1}+\operatorname{dim}_{\mathbb{k}} R_{n-2}-\operatorname{dim}_{\mathbb{k}} R_{n}=n-2
$$

for $n \geq 2$, and $\operatorname{dim}_{\mathbb{k}} M_{n}=0$ for $n<2$. Moreover, $u^{2} v=v u(u+2 v) \in M$, so $u^{2} v R \subseteq M$ and hilb $\left(u^{2} v R\right)=\operatorname{hilb} M$. So, $M=u^{2} v R$. Now

$u A \cap u v A=u M=u^{3} v R \stackrel{(*)}{=} u^{3} v A+u^{3} v^{2} A=u v u(u+2 v) A+u v u(u+2 v) v A$,

where the equality (*) holds as $R=A+v A$. Apply the map $\beta$ to the right-hand side of the equation above to yield the desired result.

We can now understand $\operatorname{ker} \lambda_{0}$ and $\operatorname{ker} \lambda_{1}$. We first prove:

Lemma 2.4. We have $\operatorname{ker} \lambda_{0}=\operatorname{ker} \lambda_{1}$.

Proof. Working in the quotient division ring of $R$, we have

$$
u^{-1} \lambda_{0}\left(e_{n}\right) u=v^{n-1} u=\lambda_{1}\left(e_{n}\right) .
$$

So for any $f \in U\left(W_{+}\right)$, we have $\lambda_{1}(f)=u^{-1} \lambda_{0}(f) u$. The result follows.

Proposition 2.5. We have that $\operatorname{ker} \lambda_{a}=\left(e_{1} e_{3}-e_{2}^{2}-e_{4}\right)$ for $a=0,1$.

Proof. We first check that $e_{1} e_{3}-e_{2}^{2}-e_{4}$ is indeed in ker $\lambda_{0}$ :

$\lambda_{0}\left(e_{1} e_{3}-e_{2}^{2}-e_{4}\right)=u\left(u v^{2}\right)-(u v)(u v)-u v^{3}=u^{2} v^{2}-u\left(u v-v^{2}\right) v-u v^{3}=0$.

Recall that $\pi_{0}=\lambda_{0} \circ \pi$. So, Lemma 2.3 implies that ker $\lambda_{0}=\pi\left(\operatorname{ker} \pi_{0}\right)$ is generated by elements $\pi(q)$ and $\pi\left(q^{\prime}\right)$ in $U\left(W_{+}\right)$. Now $\pi\left(q^{\prime}\right)=0$ by Lemma 1.1(a), so ker $\lambda_{0}$ is generated by $\pi(q)$. Moreover,

$$
\begin{aligned}
\pi(q) & =e_{1}^{2} e_{2}-e_{2} e_{1}^{2}-2 e_{2}^{2} \\
& =2\left(e_{1}\left(e_{1} e_{2}-e_{2} e_{1}\right)-e_{2}^{2}-\left(\frac{1}{2} e_{1}^{2} e_{2}-e_{1} e_{2} e_{1}+\frac{1}{2} e_{2} e_{1}^{2}\right)\right)=2\left(e_{1} e_{3}-e_{2}^{2}-e_{4}\right),
\end{aligned}
$$

using the relation $\left[e_{n}, e_{m}\right]=(m-n) e_{n+m}$ in $U\left(W_{+}\right)$. Thus, ker $\lambda_{0}=\left(e_{1} e_{3}-e_{2}^{2}-e_{4}\right)$, as claimed.

The result for $a=1$ now follows by Lemma 2.4.

It remains to analyze $\operatorname{ker} \lambda_{a}$ with $a \neq 0,1$. We do this in the next two results. 
Lemma 2.6. For $a \neq 0,1$, the kernel of $\lambda_{a}$ is generated in degrees 5 and 6 .

Proof. Take $A^{\prime}:=A(a)$. It suffices to show that the kernel of $\pi_{a}$ is generated in degrees 5 and 6 . Consider the exact sequence of right $A^{\prime}$-modules

$$
0 \longrightarrow K \longrightarrow A^{\prime}[-1] \oplus A^{\prime}[-2] \stackrel{(u,(u-a v) v)}{\longrightarrow} A^{\prime} \longrightarrow \mathbb{k} \longrightarrow 0 .
$$

We have that $u A^{\prime} \cap(u-a v) v A^{\prime} \cong K$ as right $A^{\prime}$-modules. As in the proof of Lemma 2.3, it now suffices to show that $u A^{\prime} \cap(u-a v) v A^{\prime}$ is generated in degrees 5 and 6 as a right $A^{\prime}$-module.

Let $J:=u A^{\prime} \cap(u-a v) v A^{\prime}$, and let $L:=u R \cap(u-a v) v R$. Note that $J \subseteq L$. Since $a \neq 0$, we get that $R_{\geq 2}=(u R+(u-a v) v R)_{\geq 2}$. So,

$$
\operatorname{dim}_{\mathbb{k}} L_{n}=\operatorname{dim}_{\mathbb{k}} R_{n-1}+\operatorname{dim}_{\mathbb{k}} R_{n-2}-\operatorname{dim}_{\mathbb{k}} R_{n}=n-2
$$

for $n \geq 2$. So, $\operatorname{dim}_{\mathbb{k}} L_{3}=1$, and is principally generated as a right $R$-module by an element of degree 3. In fact,

$$
L=r R, \quad \text { where } r:=u\left(u v+(1-a) v^{2}\right)=\left(u v-a v^{2}\right)(u+2 v) .
$$

Since $A_{\geq 4}^{\prime}=R_{\geq 4}$ by Proposition 2.1, we have $J_{\geq 6}=L_{\geq 6}$. By direct computation, one obtains that $J_{i}=0$ for $i=0, \ldots, 4$; one can also use Routine A.2 in the Appendix.

Let $J^{\prime}=J_{5} A^{\prime}+J_{6} A^{\prime}$. We prove by induction that $J_{n}=J_{n}^{\prime}$, for all $n \geq 5$. The statement is clear for $n=5,6$. For $n=7$, we make the following assertion, the proof of which is presented in the Appendix; see Claim A.3.

Claim. We have that $J_{5} A_{2}^{\prime} \nsubseteq J_{6} A_{1}^{\prime}$.

So for $n \geq 6$, we have $J_{n}=L_{n}=r R_{n-3}$. So $\operatorname{dim}_{\mathbb{k}} J_{7}=5$, and $\operatorname{dim}_{\mathbb{k}} J_{6} A_{1}^{\prime}=$ $\operatorname{dim}_{\mathbb{k}} J_{6}=4$. With the claim, we obtain $J_{7}=J_{5} A_{2}^{\prime}+J_{6} A_{1}^{\prime}$. Thus, $J_{7}=J_{7}^{\prime}$. Now for the induction step, suppose we have established that $J_{n}^{\prime}=J_{n}$ and $J_{n-1}^{\prime}=J_{n-1}$ for some $n \geq 7$. Then

$$
\begin{aligned}
J_{n+1} \supseteq J_{n+1}^{\prime} & =J_{n}^{\prime} u+J_{n-1}^{\prime}(u-a v) v=J_{n} u+J_{n-1}(u-a v) v \\
& =r\left(R_{n-3} u+R_{n-4}(u-a v) v\right)=r R_{n-2}=J_{n+1} .
\end{aligned}
$$

The penultimate equality holds as $a \neq 1$. Thus, the lemma is verified.

Proposition 2.8. If $a \neq 0,1$, then $\operatorname{ker} \lambda_{a}$ is the ideal generated by the elements

$$
\begin{aligned}
& h_{1}:=e_{1} e_{2}^{2}-e_{1}^{2} e_{3}-(2 a) e_{2} e_{3}+(1+2 a) e_{1} e_{4}-\left(a^{2}+a\right) e_{5}, \\
& h_{2}:=e_{1} e_{5}-4 e_{2} e_{4}+3 e_{3}^{2}+2 e_{6}, \\
& \begin{array}{r}
h_{3}:=-4 e_{1}^{2} e_{2}^{2}-4 e_{2}^{3}+4 e_{1}^{3} e_{3}+\left(20 a^{2}+14 a-7\right) e_{3}^{2} \\
\quad-\left(16 a^{2}+18 a+5\right) e_{1} e_{5}+\left(16 a^{3}+36 a^{2}+16 a-2\right) e_{6} .
\end{array}
\end{aligned}
$$


Proof. By Lemma 2.6, we just need to produce linearly independent elements of $\operatorname{ker} \lambda_{a}$ in degrees 5 and 6 . We have by Routine A.2 that $\operatorname{dim}_{\mathbb{k}}\left(\operatorname{ker} \lambda_{a}\right)_{5}=1$ and that we can choose a basis of $\left(\operatorname{ker} \lambda_{a}\right)_{5}$ to be the element $h_{1}$. In fact, we verify that $\lambda_{a}\left(h_{1}\right)=0$ using Lemma 1.3 and (1.4), while suppressing some $\mu$ superscripts:

$$
\begin{gathered}
\lambda_{a}\left(h_{1}\right)=u(u-a v) v(u-a v) v-u^{2}(u-2 a v) v^{2}-(2 a)(u-a v) v(u-2 a v) v^{2} \\
+(1+2 a) u(u-3 a v) v^{3}-\left(a^{2}+a\right)(u-4 a v) v^{4} \\
=x(x-a y)^{\mu} y(x-a y)^{\mu^{3}} y-x x^{\mu}(x-2 a y)^{\mu^{2}} y^{2} \\
-(2 a)(x-a y) y(x-2 a y)^{\mu^{2}} y^{2} \\
+(1+2 a) x(x-3 a y)^{\mu} y^{3}-\left(a^{2}+a\right)(x-4 a y) y^{4} \\
=x(x-(1+a) y) y(x-(3+a) y) y-x(x-y)(x-(2+2 a) y) y^{2} \\
-(2 a)(x-a y) y(x-(2+2 a) y) y^{2} \\
+(1+2 a) x(x-(1+3 a) y) y^{3}-\left(a^{2}+a\right)(x-4 a y) y^{4}
\end{gathered}
$$

$=0$.

On the other hand, we have by Routine A.2 that $\operatorname{dim}_{\mathbb{k}}\left(\operatorname{ker} \lambda_{a}\right)_{6}=4$ and that we can take a basis of $\left(\operatorname{ker} \lambda_{a}\right)_{6}$ to be $h_{2}, h_{3}$ along with

$$
\begin{aligned}
& h_{4}:=4 e_{2}^{3}-4 e_{1} e_{2} e_{3}+(7-4 a) e_{3}^{2}+(1+4 a) e_{1} e_{5}+\left(2-4 a-4 a^{2}\right) e_{6}, \\
& h_{5}:=4 e_{2}^{3}+(7-14 a) e_{3}^{2}-4 e_{1}^{2} e_{4}+(5+14 a) e_{1} e_{5}+\left(2-16 a-12 a^{2}\right) e_{6} .
\end{aligned}
$$

By direct computation we have

$$
\begin{aligned}
e_{1} h_{1}= & e_{1}^{2} e_{2}^{2}-e_{1}^{3} e_{3}-(2 a) e_{1} e_{2} e_{3}+(1+2 a) e_{1}^{2} e_{4}-\left(a^{2}+a\right) e_{1} e_{5}, \\
h_{1} e_{1}= & e_{1} e_{2}^{2} e_{1}-e_{1}^{2} e_{3} e_{1}-(2 a) e_{2} e_{3} e_{1}+(1+2 a) e_{1} e_{4} e_{1}-\left(a^{2}+a\right) e_{5} e_{1} \\
= & e_{1}^{2} e_{2}^{2}-e_{1}^{3} e_{3}-(2+2 a) e_{1} e_{2} e_{3}+(2 a) e_{3}^{2}+(3+2 a) e_{1}^{2} e_{4}+(4 a) e_{2} e_{4} \\
& -\left(2+7 a+a^{2}\right) e_{1} e_{5}+4\left(a^{2}+a\right) e_{6} .
\end{aligned}
$$

Claim. We have that $h_{2}, h_{3}, e_{1} h_{1}, h_{1} e_{1}$ are $\mathbb{k}$-linearly independent and that

$$
\begin{aligned}
& h_{4}=2 a(2 a+1) h_{2}-h_{3}-(6+4 a) e_{1} h_{1}+(2+4 a) h_{1} e_{1}, \\
& h_{5}=4 a^{2} h_{2}-h_{3}-(4+4 a) e_{1} h_{1}+(4 a) h_{1} e_{1} .
\end{aligned}
$$

The proof is presented in the Appendix; see Claim A.5. Thus, the result holds.

Now for the reader's convenience, we verify that $\lambda_{a}\left(h_{i}\right)=0$ for $i=2,3$ using Lemma 1.3 and (1.4), while suppressing some $\mu$ superscripts:

$$
\begin{aligned}
& \lambda_{a}\left(h_{2}\right)=u(u-4 a v) v^{4}-4(u-a v) v(u-3 a v) v^{3} \\
&+3(u-2 a v) v^{2}(u-2 a v) v^{2}+2(u-5 a v) v^{5} \\
&=x(x-4 a y)^{\mu} y^{4}-4(x-a y) y(x-3 a y)^{\mu^{2}} y^{3} \\
&+ 3(x-2 a y) y^{2}(x-2 a y)^{\mu^{3}} y^{2}+2(x-5 a y) y^{5}
\end{aligned}
$$




$$
\begin{aligned}
& =x(x-(1+4 a) y) y^{4}-4(x-a y) y(x-(2+3 a) y) y^{3} \\
& +3(x-2 a y) y^{2}(x-(3+2 a) y) y^{2}+2(x-5 a y) y^{5} \\
& =0 \text {, } \\
& \lambda_{a}\left(h_{3}\right)=-4 u^{2}(u-a v) v(u-a v) v-4(u-a v) v(u-a v) v(u-a v) v+4 u^{3}(u-2 a v) v^{2} \\
& +\left(20 a^{2}+14 a-7\right)(u-2 a v) v^{2}(u-2 a v) v^{2} \\
& -\left(16 a^{2}+18 a+5\right) u(u-4 a v) v^{4} \\
& +\left(16 a^{3}+36 a^{2}+16 a-2\right)(u-5 a v) v^{5} \\
& =-4 x x^{\mu}(x-a y)^{\mu^{2}} y(x-a y)^{\mu^{4}} y \\
& -4(x-a y) y(x-a y)^{\mu^{2}} y(x-a y)^{\mu^{4}} y+4 x x^{\mu} x^{\mu^{2}}(x-2 a y)^{\mu^{3}} y^{2} \\
& +\left(20 a^{2}+14 a-7\right)(x-2 a y) y^{2}(x-2 a y)^{\mu^{3}} y^{2} \\
& -\left(16 a^{2}+18 a+5\right) x(x-4 a y)^{\mu} y^{4} \\
& +\left(16 a^{3}+36 a^{2}+16 a-2\right)(x-5 a y) y^{5} \\
& =-4 x(x-y)(x-(2+a) y) y(x-(4+a) y) y \\
& -4(x-a y) y(x-(2+a) y) y(x-(4+a) y) y \\
& +4 x(x-y)(x-2 y)(x-(3+2 a) y) y^{2} \\
& +\left(20 a^{2}+14 a-7\right)(x-2 a y) y^{2}(x-(3+2 a) y) y^{2} \\
& -\left(16 a^{2}+18 a+5\right) x(x-(1+4 a) y) y^{4} \\
& +\left(16 a^{3}+36 a^{2}+16 a-2\right)(x-5 a y) y^{5} \\
& =0 \text {. }
\end{aligned}
$$

\section{Elementary proofs that $U\left(W_{+}\right)$and $U(W)$ are not noetherian}

In this section, we establish the remaining part of Theorem 0.7 , that ker $\lambda_{0}=$ $\operatorname{ker} \lambda_{1}$ is not finitely generated as a left or right ideal of $U\left(W_{+}\right)$. We also prove Theorem 0.8 .

We first focus on $U\left(W_{+}\right)$. Recall the map $\phi: U\left(W_{+}\right) \rightarrow B$ from Definition 0.3, and consider Notations $0.2,1.2,1.6$, and 2.2 along with the following.

Notation 3.1 $(p, I)$. Let $p:=\phi\left(e_{1} e_{3}-e_{2}^{2}-e_{4}\right)$ be an element of $B$, and let $I:=(p)$ be a two-sided ideal of $B$. Note that by Proposition 2.5, $I=\phi\left(\operatorname{ker} \lambda_{0}\right)=\pi_{B}\left(\operatorname{ker} \pi_{0}\right)$.

We begin by establishing some basic facts about $p$ and $I$.

\section{Lemma 3.2.}

(a) $p=v^{3} w-v^{2} w^{2}$.

(b) $p$ is a normal element of $S$ and of $Q$.

(c) $I=Q p$. 
Proof. We employ Lemma 1.3 and (1.4) in all parts.

(a) Consider the computation in $S$ below:

$$
\begin{aligned}
p & =\phi\left(e_{1} e_{3}-e_{2}^{2}-e_{4}\right) \\
& =u(u-2 w) v^{2}-(u-w) v(u-w) v-(u-3 w) v^{3} \\
& =x(x-2 z)^{\mu} y^{\mu^{2}} y^{\mu^{3}}-(x-z) y^{\mu}(x-z)^{\mu^{2}} y^{\mu^{3}}-(x-3 z) y^{\mu} y^{\mu^{2}} y^{\mu^{3}} \\
& =x(x-y-2 z) y^{2}-(x-z) y(x-2 y-z) y-(x-3 z) y^{3} \\
& =y^{3} z-y^{2} z^{2} \\
& =v^{3} w-v^{2} w^{2} .
\end{aligned}
$$

(b) From part (a), we get that $p$ is a normal element of $S$, and of $Q$, since $v p=p v$, $w p=p w$, and

$$
\begin{aligned}
u p & =u\left(v^{3} w-v^{2} w^{2}\right)=x y^{\mu} y^{\mu^{2}} y^{\mu^{3}} z^{\mu^{4}}-x y^{\mu} y^{\mu^{2}} z^{\mu^{3}} z^{\mu^{4}}=\left(y^{3} z-y^{2} z^{2}\right) x \\
& =\left(y^{3} z-y^{2} z^{2}\right)(x+4 y)^{\mu^{4}}=\left(v^{3} w-v^{2} w^{2}\right)(u+4 v)=p(u+4 v) .
\end{aligned}
$$

(c) On one hand, we get that $I=B p B \subseteq Q p Q=Q p$, by Lemma 1.7 and part (b). On the other hand, recall that $R$ is the subalgebra of $Q$ generated by $u, v$. We will show by induction on $i$ and $j$ that $p(v w)^{i} R_{j-2 i} \subseteq I$ for all $0 \leq i \leq\left\lfloor\frac{1}{2} j\right\rfloor$; this yields $p Q_{j} \subseteq I$.

The base case $i=j=0$ holds since $p \in I$. For the induction step, assume that $p(v w)^{i} R_{j-2 i} \subseteq I$. Now it suffices to show that (i) $p(v w)^{i} R_{j+1-2 i} \subseteq I$ and (ii) $p(v w)^{i+1} R_{j-2 i} \subseteq I$.

For (i), we have by induction that

$$
I \supseteq u p(v w)^{i} R_{j-2 i}+p(v w)^{i} R_{j-2 i} u=: I^{\prime},
$$

since $u$ is a generator of $B$. Now consider the following computations, where we suppress the action of $\mu$ on invariant elements and on graded pieces of $\mathbb{k}[x, y]$ :

$$
\begin{aligned}
I^{\prime} & =x\left(y^{3} z-y^{2} z^{2}\right)(y z)^{i} \mathbb{k}[x, y]_{j-2 i}+\left(y^{3} z-y^{2} z^{2}\right)(y z)^{i} \mathbb{k}[x, y]_{j-2 i} x^{\mu^{j+4}} \\
& =\left(y^{3} z-y^{2} z^{2}\right)(y z)^{i} x \mathbb{k}[x, y]_{j-2 i}+\left(y^{3} z-y^{2} z^{2}\right)(y z)^{i}(x+(j+4) y) \mathbb{k}[x, y]_{j-2 i} \\
& =\left(y^{3} z-y^{2} z^{2}\right)(y z)^{i}\left(x \mathbb{k}[x, y]_{j-2 i}+(x+(j+4) y) \mathbb{k}[x, y]_{j-2 i}\right) \\
& =\left(y^{3} z-y^{2} z^{2}\right)(y z)^{i} \mathbb{k}[x, y]_{j+1-2 i},
\end{aligned}
$$

where the last equality holds since $j+4>0$. Thus (i) holds.

For (ii), we get that $p(v w)^{i} R_{j+2-2 i} \subseteq I$ by applying (i) twice. Now

$$
I \supseteq p(v w)^{i} R_{j+2-2 i}+p(v w)^{i} R_{j-2 i}(u v-v w) \supseteq p(v w)^{i} R_{j-2 i}(v w) .
$$

Note that $R_{k}(v w)=(v w) R_{k}$ for all $k$. So $I \supseteq p(v w)^{i+1} R_{j-2 i}$ and we are done. 
Now we complete the proof of Theorem 0.7.

Theorem 3.3. The ideal I of $B$ is not finitely generated as a left or right ideal. As a result, the kernel of $\lambda_{0}$ is not finitely generated as a left or right ideal of $U\left(W_{+}\right)$. Proof. Recall that ker $\lambda_{0}=\left(e_{1} e_{3}-e_{2}^{2}-e_{4}\right)$ by Proposition 2.5. It is clear that if $\operatorname{ker} \lambda_{0}$ is finitely generated as a left/right ideal of $U\left(W_{+}\right)$, then $I$ is finitely generated as a left/right ideal of $B$. Therefore, to show that ker $\lambda_{0}$ is not finitely generated it suffices to show that ${ }_{B} I$ and $I_{B}$ are not finitely generated.

By way of contradiction, suppose that ${ }_{B} I$ is finitely generated. Then there exists $n \geq 4$ such that $B I_{\leq n}=I$. Since $B$ is generated by $u$ and $(u-w) v$, we get that

$$
I_{n+1}=B_{1} I_{n}+B_{2} I_{n-1}=u I_{n}+(u-w) v I_{n-1} .
$$

By Lemma 3.2, $I=Q p \subseteq S p S=S p$. Since $v I \subseteq v S p \subseteq S p$, we get by (3.4) that

$$
I_{n+1} \subseteq u S p+(u-w) S p=u S p+w S p .
$$

Using Lemma 1.3 and (1.4), it is easy to see that $u S+w S=x \llbracket[x, y, z]+z \mathbb{k}[x, y, z]$ and that a positive power of $y$ cannot belong to the right-hand side. So, a positive power of $v$ cannot belong to $u S+w S$. Therefore,

$$
v^{n-3} p \notin u S p+w S p .
$$

On the other hand, $v^{n-3} p \in I_{n+1}$ by Lemma 3.2(c). This contradicts (3.5) and (3.6). Thus, ${ }_{B} I$ is not finitely generated.

Next, suppose that $I_{B}$ is finitely generated. Then there exists $n \geq 4$ such that $I_{\leq n} B=I$, with

$$
I_{n+1}=I_{n} B_{1}+I_{n-1} B_{2}=I_{n} u+I_{n-1}(u-w) v=I_{n} u+I_{n-1} v(u+v-w) .
$$

We get that $I, I v \subseteq p S$ by Lemma 3.2(b). So, the right-hand side of (3.7) is contained in $p S u+p S(v-w)$. With an argument similar to that in the previous paragraph, we obtain that $S u+S(v-w)$ does not contain positive powers of $v$. So, $p v^{n-3} \notin I_{n} u+I_{n-1} v(u+v-w)$. On the other hand, $p v^{n-3} \in I_{n+1}$ by Lemma 3.2(b,c), which contradicts (3.7). Thus, $I_{B}$ is not finitely generated.

Remark 3.8. We do not know whether or not ker $\lambda_{a}$ is finitely generated for $a \neq 0,1$.

One can of course deduce from Theorem 3.3 that $U(W)$ and $U(V)$ are neither left nor right noetherian; see [Sierra and Walton 2014, Lemma 1.7]. Nevertheless, a direct proof that $U(W)$ is not left or right noetherian is of independent interest, and we give such a result to end the section. First, we establish some notation.

Notation 3.9 $\left(\hat{S}, \hat{R}, \widehat{B}, \widehat{\phi}, \hat{\lambda}_{a}, \eta_{a}, \widehat{I}\right)$. Since $v$ is normal in $S$ and in $R$, we may invert it. Let $\hat{S}:=S\left[v^{-1}\right]$, and let $\widehat{R}:=R\left[v^{-1}\right]$. 
Note that $\phi$ extends to an algebra homomorphism $\widehat{\phi}: U(W) \rightarrow \widehat{S}$ defined by (0.4) for all $n \in \mathbb{Z}$. Likewise, $\lambda_{a}$ extends to an algebra homomorphism $\hat{\lambda}_{a}: U(W) \rightarrow \widehat{R}$ defined by (0.5) for all $n \in \mathbb{Z}$. For $a \in \mathbb{k}$ define $\eta_{a}: \widehat{S} \rightarrow \widehat{R}$ by $u \mapsto u, v \mapsto v$, $w \mapsto a v$. Note that $\hat{\lambda}_{a}=\eta_{a} \hat{\phi}$.

Let $\widehat{B}:=\hat{\phi}(U(W))$. Finally, let $\widehat{I}=\widehat{\phi}\left(\operatorname{ker} \hat{\lambda}_{0}\right)$. Note that $\widehat{I}=\widehat{B} \cap \operatorname{ker} \eta_{0}$.

We first note that the proof of Lemma 2.4 extends to $U(W)$ to give

$$
\operatorname{ker} \hat{\lambda}_{0}=\operatorname{ker} \hat{\lambda}_{1} \text {. }
$$

Proposition 3.11. Recall $p=\phi\left(e_{1} e_{3}-e_{2}^{2}-e_{4}\right)=w(v-w) v^{2}$ from Notation 3.1 and Lemma 3.2. We have

$$
\widehat{I}=\widehat{B} \cap \operatorname{ker} \eta_{0}=\widehat{B} \cap \operatorname{ker} \eta_{1}=\widehat{B} p \widehat{B}=\widehat{S} p=p \widehat{S} .
$$

Proof. We first show that $\widehat{B} p \widehat{B}=\widehat{S} p=p \widehat{S}$. Certainly, $\widehat{B} p \widehat{B} \subseteq \widehat{S} p \widehat{S}=\widehat{S} p=p \widehat{S}$, where the last two equalities hold because a normal element of $S$ will also be normal in $\hat{S}$.

For the other direction, we will show $\widehat{R} w^{j} p \subseteq \widehat{B} p \widehat{B}$ for all $j \geq 0$ by induction. Since $\widehat{S}=\widehat{R} \cdot \mathbb{k}[w]$, this will imply $\widehat{S} p \subseteq \widehat{B} p \widehat{B}$. So assume $w^{j} p \in \widehat{B} p \widehat{B}$ for some $j \geq 0$ (it is clear for $j=0$ ). Since $u p=p(u+4 v)$, we get that for all $n \in \mathbb{Z}$,

$$
\begin{aligned}
\widehat{B} p \widehat{B} \ni\left[\hat{\phi}\left(e_{n}\right), w^{j} p\right] & =(u-(n-1) w) v^{n-1} w^{j} p-w^{j} p(u-(n-1) w) v^{n-1} \\
& =(j+4) v^{n} w^{j} p .
\end{aligned}
$$

So, $\mathbb{k}\left[v, v^{-1}\right] \cdot w^{j} p \subseteq \widehat{B} p \widehat{B}$. Since $u=\widehat{\phi}\left(e_{1}\right) \in \widehat{B}$, we have

$$
\widehat{R} w^{j} p=\mathbb{k}[u] \cdot \mathbb{k}\left[v, v^{-1}\right] \cdot w^{j} p \subseteq \widehat{B} p \widehat{B} .
$$

Finally, since we have seen that $v^{-1} w^{j} p \in \widehat{R} w^{j} p \subseteq \widehat{B} p \widehat{B}$, we have that

$$
\widehat{B} p \widehat{B} \ni\left(\hat{\phi}\left(e_{1}\right)-\widehat{\phi}\left(e_{2}\right) v^{-1}\right) w^{j} p=w^{j+1} p .
$$

By induction, $\widehat{B} p \widehat{B}=\widehat{S} p$, as desired.

From the definitions, $p \in\left(\operatorname{ker} \eta_{0}\right) \cap\left(\operatorname{ker} \eta_{1}\right)$. So

$$
\widehat{B} p \widehat{B} \subseteq\left(\operatorname{ker} \eta_{0}\right) \cap\left(\operatorname{ker} \eta_{1}\right) \cap \widehat{B}=w \hat{S} \cap(v-w) \hat{S}=w(v-w) \hat{S}=p \widehat{S} .
$$

Combining this with the first part of the proof, $\widehat{B} p \widehat{B}=\left(\operatorname{ker} \eta_{0}\right) \cap\left(\operatorname{ker} \eta_{1}\right) \cap \widehat{B}$. Then by (3.10) and the definition of $\hat{I}$, we have

$$
\widehat{I}=\left(\operatorname{ker} \eta_{0}\right) \cap \widehat{B}=\widehat{\phi}\left(\operatorname{ker} \hat{\lambda}_{0}\right)=\widehat{\phi}\left(\operatorname{ker} \hat{\lambda}_{1}\right)=\left(\operatorname{ker} \eta_{1}\right) \cap \widehat{B},
$$

completing the proof.

From Proposition 3.11 we obtain:

Theorem 3.12. The ideal $\hat{I}$ of $\widehat{B}$ is not finitely generated as a left or right ideal. As a result, the kernel of $\hat{\lambda}_{0}$ is not finitely generated as a left or right ideal of $U(W)$. 
Proof. This argument is similar to the proof of Theorem 3.3. It suffices to show that $\widehat{I}$ is not finitely generated as a left or right ideal of $\widehat{B}$.

By way of contradiction, suppose we have $\widehat{I}=\widehat{B}\left(\widehat{I}_{-n} \oplus \cdots \oplus \widehat{I}_{n}\right)$ for some $n \in \mathbb{N}$. For all $k \in \mathbb{Z}$, we have $\widehat{\phi}\left(e_{k}\right) \in u \widehat{S}+w \widehat{S}$. So, $\widehat{B}_{k} \subseteq u \widehat{S}+w \widehat{S}$ for all $k \neq 0$, and $\widehat{I}_{k} \subseteq u \hat{S}+w \hat{S}$ for all $k$ with $|k|>n$. Note that a power of $v$ cannot belong to $u \widehat{S}+w \widehat{S}$. So, $v^{n-3} p \notin \widehat{I}$. However, by Proposition 3.11, we get that $\hat{I}=\widehat{S} p$ and $v^{n-3} p \in \widehat{I}$. This contradiction shows that $\widehat{B} \hat{I}$ is not finitely generated.

The proof that $\widehat{I}_{\widehat{B}}$ is not finitely generated is similar; we leave the details to the reader.

Corollary 3.13. The universal enveloping algebra $U(V)$ is neither left nor right noetherian.

Proof. This follows directly from Theorem 3.12, since $U(W)=U(V) /(c)$.

Remark 3.14. After the first draft of this paper was finished, we learned of the results of Conley and Martin [2007]. We thank the referee for calling that work to our attention. The paper considers a family of homomorphisms defined as (using their notation)

$$
\pi_{\gamma}: U(W) \rightarrow \mathbb{k}\left[x, x^{-1}, \partial\right], \quad e_{n} \mapsto x^{n+1} \partial+(n+1) \gamma x^{n} .
$$

Using the identification $u=x^{2} \partial, v=x$ from the discussion after Theorem 0.7, we have

$$
\hat{\lambda}_{a}\left(e_{n}\right)=\left(x^{2} \partial-(n-1) a x\right) x^{n-1}=x^{n+1} \partial+(1-a)(n-1) x^{n} .
$$

The reader may verify that

$$
\hat{\lambda}_{a}(e)=x^{2(1-a)} \pi_{1-a}(e) x^{-2(1-a)}
$$

for all $e \in U(W)$ (where here one uses a suitable extension of $\mathbb{k}\left[x, x^{-1}, \partial\right]$ to carry out computations). As a result,

$$
\operatorname{ker} \hat{\lambda}_{a}=\operatorname{ker} \pi_{1-a}
$$

for all $a \in \mathbb{k}$.

Conley and Martin [2007, Theorem 1.2] showed (using (3.15)) that

$$
\operatorname{ker} \hat{\lambda}_{0}=\operatorname{ker} \hat{\lambda}_{1}=\left(e_{-1} e_{2}-e_{0} e_{1}-e_{1}\right) \text {. }
$$

Recall from Proposition 2.5 that ker $\lambda_{0}$ is generated as a two-sided ideal by $g_{4}:=$ $e_{1} e_{3}-e_{2}^{2}-e_{4}$. A computation gives that

$$
\operatorname{ad}\left(e_{-1}^{3}\right)\left(g_{4}\right)=\left[e_{-1},\left[e_{-1},\left[e_{-1}, g_{4}\right]\right]\right]=12\left(e_{-1} e_{2}-e_{0} e_{1}-e_{1}\right),
$$

and it follows that

$$
\left(g_{4}\right)=\operatorname{ker} \hat{\lambda}_{0}=\operatorname{ker} \hat{\lambda}_{1}=\left(e_{-1} e_{2}-e_{0} e_{1}-e_{1}\right) .
$$




\section{The connection between the maps $\phi$ and $\rho$}

For the remainder of the paper, we return to considering $U\left(W_{+}\right)$. The main goal of this section is to relate the map $\phi$ (of Definition 0.3) that played a crucial role in the proof of Theorem 3.3 to the map $\rho$ (of Notation 0.9) that was the focus of [Sierra and Walton 2014]. We show that $\operatorname{ker} \phi=\operatorname{ker} \rho$; in fact, we have the next result.

Theorem 4.1. We have that $\operatorname{ker} \rho=\operatorname{ker} \phi=\bigcap_{a \in \mathbb{k}} \operatorname{ker} \lambda_{a}$. As a consequence, $\rho\left(U\left(W_{+}\right)\right) \cong \phi\left(U\left(W_{+}\right)\right)$.

Consider Notation 0.2 and the following notation for this section. Recall the definitions of $X, f, \tau$ from Notation 0.9. So, $\tau \in \operatorname{Aut}(X)$ and $\tau^{*}: \mathbb{k}(X) \rightarrow \mathbb{k}(X)$ is the pullback of $\tau$. Here we take $\mu \in \operatorname{Aut}\left(\mathbb{P}^{2}\right)$ and $v \in \operatorname{Aut}\left(\mathbb{P}^{1}\right)$ to be morphisms of varieties, defined by

$$
\mu([x: y: z])=[x-y: y: z] \text { and } \quad v([x: y])=[x-y: y] .
$$

We denote the respective pullback morphisms by $\mu^{*}$ and $v^{*}$. However, to be consistent with Lemma 1.3 (and abusing notation slightly), we still write

$$
S \cong \mathbb{k}[x, y, z]^{\mu} \text { and } R \cong \mathbb{k}[x, y]^{\nu} .
$$

We also establish the convention that $h^{\tau}:=\tau^{*} h$ for $h \in \mathbb{k}(X)$, and similarly for pullback by other morphisms.

Before proving Theorem 4.1, we provide some preliminary results.

Lemma 4.2 $\left(\psi_{a}, \Psi_{a}\right)$. For $a \in \mathbb{k}$, we have the following statements.

(a) We have a well-defined morphism $\psi_{a}: \mathbb{P}^{1} \rightarrow X$ given by

$\psi_{a}([x: y])=\left[2 x^{2}-4 x y-6 a y^{2}: x^{2}-2 x y+y^{2}:-x^{2}+3 x y-2 y^{2}: x^{2}-4 x y+4 y^{2}\right]$.

(b) $\psi_{a} v=\tau \psi_{a}$.

(c) $\psi_{a}^{*}$ extends to an algebra homomorphism $\Psi_{a}: \mathbb{k}(X)\left[t ; \tau^{*}\right] \rightarrow \mathbb{k}\left(\mathbb{P}^{1}\right)\left[s ; v^{*}\right]$, where $\Psi_{a}(t)=s$.

Proof. (a,b) Both are straightforward. Part (a) is a direct computation. On page 508 in the Appendix, we verify that $\left(\psi_{a} v\right)^{*}=v^{*} \psi_{a}^{*}=\psi_{a}^{*} \tau^{*}=\left(\tau \psi_{a}\right)^{*}$ as maps from $\mathbb{k}(X) \rightarrow \mathbb{k}\left(\mathbb{P}^{1}\right)$. Thus, (b) holds.

(c) We have for all $h, \ell \in \mathbb{k}(X)$ and $n, m \in \mathbb{N}$ that

$$
\begin{aligned}
\Psi_{a}\left(h t^{n} \ell t^{m}\right) & =\Psi_{a}\left(h \ell^{\tau^{n}} t^{n+m}\right)=\psi_{a}^{*}(h) \psi_{a}^{*}\left(\ell^{\tau^{n}}\right) s^{n+m} \\
& =\psi_{a}^{*}(h) \psi_{a}^{*}(\ell)^{v^{n}} s^{n+m}=\psi_{a}^{*}(h) s^{n} \psi_{a}^{*}(\ell) s^{m}=\Psi_{a}\left(h t^{n}\right) \Psi_{a}\left(\ell t^{m}\right) .
\end{aligned}
$$

Thus, $\Psi_{a}$ is an algebra homomorphism. 
Lemma $4.3\left(C_{a}\right)$. For $a \in \mathbb{k}$, define the curve

$$
C_{a}=V\left(w+6 a x+(4+12 a) y+(2+6 a) z, x z-y^{2}\right) \subseteq X .
$$

Then $\psi_{a}$ defines an isomorphism from $\mathbb{P}^{1} \rightarrow C_{a}$.

Proof. That the image of $\psi_{a}$ of Lemma 4.2(a) is contained in $C_{a}$ is a straightforward verification. The inverse map to $\psi_{a}$ is defined by the birational map $[w: x: y: z] \mapsto$ $[2 x+y: x+y]$; we leave the verification of the details to the reader.

Lemma 4.4 $(\gamma)$. Define a map $\gamma: R \rightarrow \mathbb{k}\left(\mathbb{P}^{1}\right)\left[s ; v^{*}\right]$ as follows: if $h \in R_{n}=\mathbb{k}[x, y]_{n}$, let

$$
\gamma(h)=\frac{h}{x(x-y) \cdots(x-(n-1) y)} s^{n} .
$$

Then $\gamma$ is an injective $\mathbb{k}$-algebra homomorphism.

Proof. Let $h \in \mathbb{k}[x, y]_{n}$ and $\ell \in \mathbb{k}[x, y]_{m}$. Then

$$
\begin{aligned}
\gamma(h * \ell) & =\gamma\left(h \ell^{\nu^{n}}\right)=\frac{h \ell^{\nu^{n}}}{x(x-y) \cdots(x-(n+m-1) y)} s^{n+m} \\
& =\frac{h}{x(x-y) \cdots(x-(n-1) y)}\left(\frac{\ell}{x(x-y) \cdots(x-(m-1) y)}\right)^{v^{n}} s^{m+n} \\
& =\frac{h}{x(x-y) \cdots(x-(n-1) y)} s^{n} \frac{\ell}{x(x-y) \cdots(x-(m-1) y)} s^{m}=\gamma(h) \gamma(\ell) .
\end{aligned}
$$

So, $\gamma$ is a homomorphism; injectivity is clear.

Proposition 4.5. Retain the notation of Lemmas 4.2 and 4.4. Let $a \in \mathbb{k}$. Then $\Psi_{a} \rho=\gamma \lambda_{a}$ as maps from $U\left(W_{+}\right) \rightarrow \mathbb{k}\left(\mathbb{P}^{1}\right)\left[s ; v^{*}\right]$, and $\operatorname{ker} \Psi_{a} \rho=\operatorname{ker} \lambda_{a}$.

Proof. By Lemma 1.1(a), it suffices to verify that the maps $\Psi_{a} \rho$ and $\gamma \lambda_{a}$ agree on $e_{1}$ and $e_{2}$. We have

$$
\Psi_{a}\left(\rho\left(e_{1}\right)\right)=\Psi_{a}(t)=s=\gamma(u)=\gamma\left(\lambda_{a}\left(e_{1}\right)\right) .
$$

We verify that

$$
\psi_{a}^{*}(f)=\frac{x y-a y^{2}}{x^{2}-x y}
$$

on page 508 in the Appendix. Thus,

$$
\Psi_{a}\left(\rho\left(e_{2}\right)\right)=\psi_{a}^{*}(f) s^{2}=\frac{x y-a y^{2}}{x^{2}-x y} s^{2}=\gamma\left(u v-a v^{2}\right)=\gamma \lambda_{a}\left(e_{2}\right) .
$$

The final statement follows from the fact that $\gamma$ is injective (Lemma 4.4). 
Proof of Theorem 4.1. By Lemma 4.3, $\psi_{a}^{*} h=0$ if and only if $\left.h\right|_{C_{a}} \equiv 0$. Now, the curves $C_{a}$ cover an open subset of $X$. (One way to see this is that, because $\bigcup_{a} C_{a}$ is dense in $X$ and is clearly constructible, by [Hartshorne 1977, Exercise II.3.19(b)] it contains an open subset of $X$.) Thus if $h \in \mathbb{k}(X)$ is in the intersection $\bigcap_{a} \operatorname{ker} \psi_{a}^{*}$, then $h$ vanishes on this open subset and so $h=0$. So, $\bigcap_{a} \operatorname{ker} \Psi_{a}=\{0\}$. Thus, $\operatorname{ker} \rho=\bigcap_{a} \operatorname{ker} \Psi_{a} \rho=\bigcap_{a} \operatorname{ker} \lambda_{a}$, where the last equality holds by Proposition 4.5.

To show that $\operatorname{ker} \phi=\bigcap_{a} \operatorname{ker} \lambda_{a}$, define closed immersions $i_{a}: \mathbb{P}^{1} \rightarrow \mathbb{P}^{2}$ for $a \in \mathbb{k}$ by $i_{a}([x: y])=[x: y: a y]$. Then $\operatorname{im}\left(i_{a}\right)=V(z-a y)$, and pullback along $i_{a}$ induces the ring homomorphism

$$
i_{a}^{*}: \mathbb{k}[x, y, z] \rightarrow \mathbb{k}[x, y], \quad x \mapsto x, y \mapsto y, z \mapsto a y .
$$

The reader may verify that $i_{a} v=\mu i_{a}$, and that $i_{a}^{*}$ is also a homomorphism from $S=\mathbb{k}[x, y, z]^{\mu}$ to $R=\mathbb{k}[x, y]^{\nu}$. In terms of $u, v, w$, we have

$$
i_{a}^{*}(u)=u, \quad i_{a}^{*}(v)=v, \quad i_{a}^{*}(w)=a v .
$$

That is, $i_{a}^{*}=\left.\eta_{a}\right|_{S}$, where $\eta_{a}$ was defined in Notation 3.9. We see that $i_{a}^{*} \phi=\lambda_{a}$.

As with the first paragraph, the curves $V(z-a y)$ cover an open subset of $\mathbb{P}^{2}$ : in fact, $\bigcup_{a} V(z-a y) \supseteq\left(\mathbb{P}^{2} \backslash V(y)\right)$. So $\bigcap_{a} \operatorname{ker} i_{a}^{*}=\{0\}$. Thus, $\operatorname{ker} \phi=\bigcap_{a} \operatorname{ker} i_{a}^{*} \phi=$ $\bigcap_{a} \operatorname{ker} \lambda_{a}$, completing the proof.

\section{The kernel of $\phi$}

In this section, we analyze the map $\phi$ from Definition 0.3 . In particular, we verify part (c) of Theorem 0.6. To proceed, recall Notations 0.2, 1.2, 1.6, and 2.2.

Theorem 5.1. The kernel of $\phi$ is generated as a two-sided ideal by

$$
g:=e_{1} e_{5}-4 e_{2} e_{4}+3 e_{3}^{2}+2 e_{6} .
$$

Proof. First, observe that as $e_{1} e_{5}, e_{2} e_{4}, e_{3}^{2}, e_{6}$ are elements of the standard basis for $U\left(W_{+}\right)$(by Lemma 1.1(b)), they are linearly independent. So, we have that $g \neq 0$.

Now we verify that $\phi(g)=0$ by using Lemma 1.3 and (1.4):

$$
\begin{aligned}
\phi(g)= & u(u-4 w) v^{4}-4(u-w) v(u-3 w) v^{3}+3(u-2 w) v^{2}(u-2 w) v^{2}+2(u-5 w) v^{5} \\
= & x(x-4 z)^{\mu} y^{4}-4(x-z) y(x-3 z)^{\mu^{2}} y^{3} \\
& \quad+3(x-2 z) y^{2}(x-2 z)^{\mu^{3}} y^{2}+2(x-5 z) y^{5} \\
= & x(x-y-4 z) y^{4}-4(x-z) y(x-2 y-3 z) y^{3} \\
& +3(x-2 z) y^{2}(x-3 y-2 z) y^{2}+2(x-5 z) y^{5} \\
= & 0 .
\end{aligned}
$$

We take the following notation for the rest of the proof. 
Notation $5.2\left(M, M^{\prime}, b_{5}, b_{6}, b_{7}, \eta\right)$. Consider the right $B$-modules

$$
M:=u B \cap(u-w) v B \text { and } \quad M^{\prime}:=b_{5} B+b_{6} B+b_{7} B,
$$

with

$$
\begin{aligned}
& b_{5}=(u v-v w)\left(u^{3}-6(u v-v w) u+12 u(u v-v w)\right), \\
& b_{6}=(u v-v w)\left(-48(u v-3 v w) v^{2}-36 u(u v-2 v w) v+u^{4}\right), \\
& b_{7}=(u v-v w)\left(u^{5}-40\left((u v-v w)^{2} u-3(u v-v w) u(u v-v w)+3 u(u v-v w)^{2}\right)\right) .
\end{aligned}
$$

Further, take $\eta: B \rightarrow A(0)$ to be the map induced by the projection $\eta_{0}: \widehat{S} \rightarrow \widehat{R}=$ $\widehat{S} /(w)$ from Notation 3.9.

The remainder of the proof will be established through a series of lemmas.

Lemma 5.3. We obtain that $b_{5}, b_{6}, b_{7} \in u B \cap(u-w) v B$. In other words, $M^{\prime} \subseteq M$. Proof. Let

$$
\begin{aligned}
& r_{5}:=e_{2}\left(e_{1}^{3}-6 e_{2} e_{1}+12 e_{1} e_{2}\right), \\
& r_{6}:=e_{2}\left(-48 e_{4}-36 e_{1} e_{3}+e_{1}^{4}\right), \\
& r_{7}:=e_{2}\left(e_{1}^{5}-40\left(e_{2}^{2} e_{1}-3 e_{2} e_{1} e_{2}+3 e_{1} e_{2}^{2}\right)\right) .
\end{aligned}
$$

We have as a consequence of the degree-5 relation of $U\left(W_{+}\right)$in Lemma 1.1(a) that

$$
r_{5}=e_{1}\left(e_{1}^{2} e_{2}-3 e_{1} e_{2} e_{1}+3 e_{2} e_{1}^{2}+6 e_{2}^{2}\right),
$$

and as a consequence of the degree-7 relation of $U\left(W_{+}\right)$in Lemma 1.1(a) that

$$
r_{7}=e_{1}\left(e_{1}^{4} e_{2}-5 e_{1}^{3} e_{2} e_{1}+10 e_{1}^{2} e_{2} e_{1}^{2}-10 e_{1} e_{2} e_{1}^{3}+5 e_{2} e_{1}^{4}-40 e_{2}^{3}\right) .
$$

Thus $r_{5}, r_{7} \in e_{1} U\left(W_{+}\right) \cap e_{2} U\left(W_{+}\right)$. Since $b_{5}=\phi\left(r_{5}\right)$ and $b_{7}=\phi\left(r_{7}\right)$, these are both in $u B \cap(u v-v w) B$.

Note that $r_{6} \in e_{2} U\left(W_{+}\right)$, so $b_{6}=\phi\left(r_{6}\right) \in(u-w) v B$. Further,

$$
r_{6}=e_{1}\left(-36 e_{2} e_{3}-18 e_{5}+2 e_{4} e_{1}-e_{3} e_{1}^{2}+e_{2} e_{1}^{3}\right)+12 g .
$$

Thus, $b_{6} \in u B$ as well.

Lemma 5.9. Suppose that $M=M^{\prime}$. Then $\operatorname{ker} \phi=(g)$ and the theorem holds.

Proof. Let $K$ be the kernel of

$$
\alpha: B[-1] \oplus B[-2] \rightarrow B, \quad\left(b, b^{\prime}\right) \mapsto\left(u b+(u v-v w) b^{\prime}\right) .
$$

It is a standard fact that the map

$$
\beta: M \rightarrow K
$$


defined by $\beta(r)=\left(u^{-1} r,-(u v-v w)^{-1} r\right)$ is an isomorphism of graded right $B$-modules, as in the proof of Lemma 2.3. Thus, $K$ is generated by $\beta\left(b_{5}\right), \beta\left(b_{6}\right)$, and $\beta\left(b_{7}\right)$ by the assumption. By Proposition A.1 in the Appendix, the kernel of $\pi_{B}$ is generated as a two-sided ideal of $\mathbb{k}\left\langle t_{1}, t_{2}\right\rangle$ by a degree-5 element $q_{5}$, a degree- 6 element $q_{6}$, and a degree- 7 element $q_{7}$. We compute $q_{5}$ and $q_{7}$ by applying the formula from Proposition A.1 to $\beta\left(b_{5}\right)$ and $\beta\left(b_{7}\right)$, and by using (5.4)-(5.8). Namely, take

$$
\begin{aligned}
& \tilde{b}_{1}^{1}=t_{1}^{2} t_{2}-3 t_{1} t_{2} t_{1}+3 t_{2} t_{1}^{2}+6 t_{2}^{2}, \\
& \tilde{b}_{2}^{1}=-t_{1}^{3}+6 t_{2} t_{1}-12 t_{1} t_{2}, \\
& \tilde{b}_{1}^{2}=t_{1}^{4} t_{2}-5 t_{1}^{3} t_{2} t_{1}+10 t_{1}^{2} t_{2} t_{1}^{2}-10 t_{1} t_{2} t_{1}^{3}+5 t_{2} t_{1}^{4}-40 t_{2}^{3}, \\
& \tilde{b}_{2}^{2}=-t_{1}^{5}+40\left(t_{2}^{2} t_{1}-3 t_{2} t_{1} t_{2}+3 t_{1} t_{2}^{2}\right) .
\end{aligned}
$$

So, we have that

$$
\begin{aligned}
& q_{5}=t_{1} \tilde{b}_{1}^{1}+t_{2} \tilde{b}_{2}^{1}=\left[t_{1},\left[t_{1},\left[t_{1}, t_{2}\right]\right]\right]+6\left[t_{2},\left[t_{2}, t_{1}\right]\right], \\
& q_{7}=t_{1} \tilde{b}_{1}^{2}+t_{2} \tilde{b}_{2}^{2}=\left[t_{1},\left[t_{1},\left[t_{1},\left[t_{1},\left[t_{1}, t_{2}\right]\right]\right]\right]\right]+40\left[t_{2},\left[t_{2},\left[t_{2}, t_{1}\right]\right]\right] .
\end{aligned}
$$

By Lemma 1.1(a), $q_{5}$ and $q_{7}$ generate the kernel of $\pi$. So, $\operatorname{ker} \phi=\pi\left(\operatorname{ker} \pi_{B}\right)=$ $\left(\pi\left(q_{6}\right)\right)$. We see immediately that $(\operatorname{ker} \phi)_{6}$ is a 1 -dimensional $\mathbb{k}$-vector space, generated by $\pi\left(q_{6}\right)$. Since $g \in(\operatorname{ker} \phi)_{6}$ is nonzero, we have $g=\pi\left(q_{6}\right)$ up to a scalar multiple. Therefore, $\operatorname{ker} \phi=(g)$.

Our goal now is to show that $M=M^{\prime}$; we do this by comparing Hilbert series.

Lemma 5.10. The Hilbert series of $M$ is $t^{5}(1-t)^{-2}\left(1-t^{2}\right)^{-1}$.

Proof. Since $A(0)=\mathbb{k} \oplus u R$ we have

$$
\text { hilb } A(0)=1+t(\text { hilb } R)=1+\frac{t}{(1-t)^{2}}=\frac{1-t+t^{2}}{(1-t)^{2}} .
$$

On the other hand, it is well known that

$$
\text { hilb } Q=\operatorname{hilb} \mathbb{k}[x, y, y z]=\frac{1}{(1-t)^{2}\left(1-t^{2}\right)} .
$$

Since $\lambda_{0}=\eta \circ \phi$, we get that $\operatorname{ker} \eta=\phi\left(\operatorname{ker} \lambda_{0}\right)$ (which is denoted by $I$ in Notation 3.1). So, by Lemma 3.2(c), we get

$$
\text { hilb ker } \eta=\frac{t^{4}}{(1-t)^{2}\left(1-t^{2}\right)} \text {. }
$$


Then

hilb $B=$ hilb $A(0)+$ hilb ker $\eta$

$$
=\frac{1-t+t^{3}-t^{4}}{(1-t)^{2}\left(1-t^{2}\right)}+\frac{t^{4}}{(1-t)^{2}\left(1-t^{2}\right)}=\frac{1-t+t^{3}}{(1-t)^{2}\left(1-t^{2}\right)} .
$$

Finally, we compute hilb $M$ from the exact sequence

$$
0 \longrightarrow M \stackrel{\beta}{\longrightarrow} B[-1] \oplus B[-2] \stackrel{\alpha}{\longrightarrow} B \longrightarrow \mathbb{k} \longrightarrow 0,
$$

where $\alpha, \beta$ are as in the proof of Lemma 5.9. This gives

$$
\text { hilb } M=\left(t^{2}+t-1\right)(\text { hilb } B)+1=\frac{t^{5}}{(1-t)^{2}\left(1-t^{2}\right)},
$$

as claimed.

We now provide results on the Hilbert series of $M^{\prime}$.

Lemma 5.11. We have that hilb $\eta\left(M^{\prime}\right) \geq t^{5}(1-t)^{-2}$.

Proof. Let $a_{5}:=\eta\left(b_{5}\right)$ and $a_{6}:=\eta\left(b_{6}\right)$. Then

$$
\begin{aligned}
a_{5} & =u v u\left(u^{2}-6 v u+12 u v\right) \\
& =x y(x-2 y)((x-3 y)(x-4 y)-6 y(x-4 y)+12(x-3 y) y) \\
& =x^{2}(x-y)(x-2 y) y, \\
a_{6} & =u v u\left(u^{3}-36 u v^{2}-48 v^{3}\right) \\
& =x y(x-2 y)\left((x-3 y)(x-4 y)(x-5 y)-36(x-3 y) y^{2}-48 y^{3}\right) \\
& =x^{2}(x-y)(x-2 y) y(x-11 y) \\
& =a_{5}(u-6 v) .
\end{aligned}
$$

Since $a_{5} u$ and $a_{5}(u-6 v)$ are in $\eta\left(M^{\prime}\right)$ and $u$ and $u-6 v$ span $R_{1}$, we have $a_{5} R_{1} \subseteq$ $\eta\left(M^{\prime}\right)$. We get that $\eta\left(M^{\prime}\right) \supseteq a_{5} A(0)+a_{5} R_{1} A(0)$, as $\eta\left(M^{\prime}\right)$ is a right $A(0)$-module and contains $a_{5} R_{\leq 1}$. Since $A(0)+R_{1} A(0)=R$, we obtain that $\eta\left(M^{\prime}\right) \supseteq a_{5} R$. Now as hilb $R=(1-t)^{-2}$, we conclude that hilb $\eta\left(M^{\prime}\right) \geq t^{5}(1-t)^{-2}$.

Lemma 5.12. We have that $\operatorname{hilb}\left(M^{\prime} \cap \operatorname{ker} \eta\right) \geq t^{7}(1-t)^{-2}\left(1-t^{2}\right)^{-1}$.

Proof. Again, recall that $\operatorname{ker} \eta=\phi\left(\operatorname{ker} \lambda_{0}\right)$, which is denoted by $I$ in Notation 3.1. Moreover by Lemma 3.2(c), we have $I=Q p=p Q$, where $p=v^{3} w-v^{2} w^{2}$. Let

$$
h:=(u v-v w)(u+2 v) p=(x y-y z) x\left(y^{3} z-y^{2} z^{2}\right) .
$$

Claim. We have

$b_{5} Q+b_{6} Q+b_{7} Q \ni x(x y-y z)\left(x y z+y^{2} z\right)=(u v-v w)(u+2 v)(u+4 v) v w$. 
The proof of this claim is provided in the Appendix; see Claim A.6(a).

Since $M^{\prime} \cap I \supseteq M^{\prime} I=b_{5} Q p+b_{6} Q p+b_{7} Q p$, we have

$$
\begin{aligned}
M^{\prime} \cap I & \supseteq(u v-v w)(u+2 v)(u+4 v) v w p Q \\
& =(x y-y z) x\left(y^{3} z-y^{2} z^{2}\right)(x+y) y z Q=h(x+y) y z Q .
\end{aligned}
$$

We now show by induction that $M^{\prime} \cap I \supseteq h Q_{n}$ for all $n \geq 0$.

Claim. $M^{\prime} \cap I \supseteq h Q_{n}$ for $n=0,1,2$.

The proof of this assertion is provided in the Appendix; see Claim A.6(b). We will prove the result for larger $n$ by geometric arguments. The maximal graded nonirrelevant ideals of $\mathbb{k}[x, y, y z]$ are in bijective correspondence with $\mathbb{k}$-points of the weighted projective plane $\mathbb{P}(1,1,2)$ [Harris 1992, Example 10.27]. We use the notation $(a: b: c)$ to denote a point of $\mathbb{P}(1,1,2)$. Let

$$
K(n):=(x-n y) \mathbb{k}[x, y, y z]+\left(y^{2}-y z\right) \mathbb{k}[x, y, y z]
$$

be the graded ideal of polynomials vanishing at $(n: 1: 1)$.

Suppose now that $M^{\prime} \cap I \supseteq h Q_{n}$ for some $n \geq 2$. Then $M^{\prime} \cap I$ contains

$$
\begin{aligned}
h\left(Q_{n} u\right. & \left.+Q_{n-1}(u v-v w)\right) \\
= & h((x-(n+7) y) \mathbb{k}[x, y, y z]+((x-(n+6) y) y-y z) \mathbb{k}[x, y, y z])_{n+1} \\
= & h\left((x-(n+7) y) \mathbb{k}[x, y, y z]+\left(y^{2}-y z\right) \mathbb{k}[x, y, y z]\right)_{n+1} \\
& =h K(n+7)_{n+1} .
\end{aligned}
$$

From (5.13), we get $\left(M^{\prime} \cap I\right)_{n+1} \ni h\left(x y z+y^{2} z\right) y^{n-2}$. Since $\left(x y z+y^{2} z\right) y^{n-2}$ does not vanish at $(n+7: 1: 1)$, it is not in $h K(n+7)_{n+1}$. Thus,

$$
h K(n+7)_{n+1}+\mathbb{k} h\left(x y z+y^{2} z\right) y^{n-2}=h \mathbb{k}[x, y, y z]_{n+1} \subseteq M^{\prime} \cap I,
$$

where the equality holds as $h K(n+7)_{n+1}$ is codimension 1 in $h \llbracket[x, y, y z]_{n+1}$. Hence, $h Q_{n+1} \subseteq M^{\prime} \cap I$.

Now, by induction, we obtain $M^{\prime} \cap I \supseteq h Q$. Since hilb $Q=(1-t)^{-2}\left(1-t^{2}\right)^{-1}$, we have

$$
\operatorname{hilb}\left(M^{\prime} \cap I\right) \geq \frac{t^{7}}{(1-t)^{2}\left(1-t^{2}\right)} .
$$

Lemma 5.14. We have that hilb $M=$ hilb $M^{\prime}=t^{5}(1-t)^{-2}\left(1-t^{2}\right)^{-1}$. As a result, $M=M^{\prime}$.

Proof. Combining Lemmas 5.11 and 5.12, we have

$$
\operatorname{hilb}\left(M^{\prime}\right) \geq \frac{t^{5}}{(1-t)^{2}}+\frac{t^{7}}{(1-t)^{2}\left(1-t^{2}\right)}=\frac{t^{5}}{(1-t)^{2}\left(1-t^{2}\right)} .
$$


On the other hand, by Lemmas 5.3 and 5.10,

$$
\operatorname{hilb}\left(M^{\prime}\right) \leq \frac{t^{5}}{(1-t)^{2}\left(1-t^{2}\right)} .
$$

Thus, hilb $M=$ hilb $M^{\prime}$. Since $M^{\prime} \subseteq M$ again by Lemma 5.3, we conclude that $M=M^{\prime}$.

Theorem 5.1 now follows from Lemmas 5.9 and 5.14.

Remark 5.15. A shorter proof of Theorem 5.1 follows from the results of [Conley and Martin 2007]. Recall from Notation 3.9 that we may extend $\phi$ to a map $\widehat{\phi}: U(W) \rightarrow \widehat{S}$, using the same formula $(0.4)$ for $\widehat{\phi}\left(e_{n}\right)$ with $n \leq 0$. Then [Conley and Martin 2007, Theorem 1.3] and (3.15), together with Theorem 4.1, give that ker $\widehat{\phi}=\left(e_{-1} e_{3}-4 e_{0} e_{2}+3 e_{1}^{2}+2 e_{2}\right)$. The reader may verify that

$$
\operatorname{ad}\left(e_{-1}^{4}\right)(g)=\left[e_{-1},\left[e_{-1},\left[e_{-1},\left[e_{-1}, g\right]\right]\right]\right]=24\left(e_{-1} e_{3}-4 e_{0} e_{2}+3 e_{1}^{2}+2 e_{2}\right) .
$$

Since $\widehat{\phi}(g)=0$, we have $(g) \subseteq \operatorname{ker} \widehat{\phi}=\left(e_{-1} e_{3}-4 e_{0} e_{2}+3 e_{1}^{2}+2 e_{2}\right) \subseteq(g)$, so all are equal.

\section{A partial result on chains of two-sided ideals}

It is not known whether $U\left(W_{+}\right)$satisfies the ascending chain condition (ACC) on two-sided ideals; see Question 0.11. We do not answer this question here; however, we prove the partial result that the non-noetherian factor $B$ of $U\left(W_{+}\right)$does have ACC on two-sided ideals.

Recall Notations 0.2,1.2, 1.6; in particular, $Q$ is the subalgebra of $S$ generated by $u, v, v w$. Throughout, we consider $B$ as a subalgebra of $Q$. We begin by proving:

Lemma 6.1. Let $h$ be a nonzero, homogeneous, normal element of $Q$, and let $a \in \mathbb{k}$. Then the Q-bimodules

$$
N:=h Q / h v Q \quad \text { and } \quad M_{a}=h Q / h\left(v w-a v^{2}\right) Q
$$

are noetherian $B$-bimodules under the action induced from $Q$.

Proof. We remark that any normal element of $Q$ must be in the commutative subalgebra $\mathbb{k}[v, v w]$, and thus, must commute with $v$ and $v w$. In particular, $v Q N=0$ and $\left(v w-a v^{2}\right) Q M_{a}=0=M_{a}\left(v w-a v^{2}\right) Q$.

Let $\theta: Q \rightarrow Q / v Q$ be the canonical projection. (Note that $v w \notin \operatorname{ker} \theta$.) Since $u(v w)-(v w) u=2 v^{2} w$ is contained in $\operatorname{ker} \theta$, the image $Q / v Q$ is commutative. It is easy to see that $Q / v Q \cong \mathbb{k}[s, t]$ under the identification $s=\theta(u), t=\theta(v w)=$ $\theta(u v-v w)$. Note that $s=\theta\left(\phi\left(e_{1}\right)\right)$ and $t=\theta\left(\phi\left(e_{2}\right)\right)$ are in $B$. So, $\theta(B)=Q / v Q$. Thus, a left $B$-submodule of $h Q / h v Q$ is simply an ideal of $\mathbb{k}[s, t]$. So, $h Q / h v Q$ 
is noetherian as a left $B$-module. As chains of $B$-bimodules are also chains of left $B$-modules, $h Q / h v Q$ is also a noetherian $B$-bimodule.

Now define an algebra homomorphism $\delta: Q \rightarrow R$ by $\delta(u)=u, \delta(v)=v$, and $\delta(v w)=a v^{2}$. (Note that $\delta=\left.\eta_{a}\right|_{Q}$ from Notation 3.9.) It is easy to see that $\operatorname{ker} \delta=\left(v w-a v^{2}\right) Q$ and that $\delta$ is surjective. Note also that $\delta\left(\phi\left(e_{1}\right)\right)=u$ and $\delta\left(\phi\left(e_{2}\right)\right)=u v-a v^{2}$. Thus, $\delta(B)=A(a)$ as subalgebras of $R$. If $a \neq 0,1$, then by Proposition 2.1, $A(a) \supseteq R_{\geq 4}$ is noetherian, and $R$ is a finitely generated right $A(a)$-module. If $a=0$, then $R=A(0)+v A(0)$ is again a finitely generated right $A(0)$-module, and $A(0)$ is noetherian. Thus for $a \neq 1, M_{a}$ is also a finitely generated right $A(a)$-module. So, $M_{a}$ is noetherian as a right $B$-module, let alone a $B$-bimodule.

If $a=1$ then we have, similarly, that $\delta(B)=A(1)$ is noetherian, and that $R=A(1)+A(1) v$ is a finitely generated left $A(1)$-module. It follows that $M_{a}$ is a finitely generated left $A(a)$-module. So, $M_{a}$ is noetherian as a left $B$-module, and again as a $B$-bimodule.

We now use geometric arguments to show:

Proposition 6.2. Suppose that $\mathbb{k}$ is algebraically closed, and let $K \subseteq Q$ be a nonzero graded ideal. Then $Q / K$ is a noetherian B-bimodule.

Proof. Let $T$ be the commutative ring $\mathbb{k}[x, y, y z]$. We consider $K$ as a subset of $T$, since (via Lemma 1.3) $Q=T^{\mu}$ and $T$ have the same underlying vector space. For all $n, m \in \mathbb{N}$, we have

$$
K_{n+m} \supseteq K_{n} Q_{m}=K_{n}\left(T_{m}\right)^{\mu^{n}}=K_{n} T_{m},
$$

and so $K$ is also an ideal of $T$. Further,

$$
K_{n+m} \supseteq Q_{m} K_{n}=T_{m}\left(K_{n}\right)^{\mu^{m}} .
$$

If $T$ were generated in degree 1, one could obtain directly from (6.3) and (6.4) that $K_{n}$ is $\mu$-invariant for $n \gg 0$ (or see [Artin and Stafford 1995, Lemma 4.4]). A similar statement holds in our case; however, a proof would take us too far afield so we work more directly with the graded pieces of $K$.

Choose $n_{0}$ so that $K_{n_{0}} \neq 0$. For all $n \geq n_{0}$, let $h_{n} \neq 0$ be a greatest common divisor of $K_{n}$, considered as a subset of $T_{n}$. By (6.3), $h_{n+1} \mid h_{n} x, h_{n} y$. Since $x, y$ have no common divisor, we have $h_{n+1} \mid h_{n}$ for all $n \geq n_{0}$. This chain of divisors must stabilize, and thus there is $n_{1} \geq n_{0}$ such that $h_{n+1} h_{n}^{-1} \in \mathbb{k}$ for $n \geq n_{1}$. Let $h:=h_{n_{1}}$.

By (6.4), $h \mid \mu^{m}(h)$ for all $m \in \mathbb{N}$, so $h$ is an eigenvector of $\mu$. Thus, $h$ is normal in $Q$. Since $h \mid f$ for all $f \in K$, we can write $K=h J$ for some $J \subseteq Q$. Since $h$ is normal, $J$ is again an ideal of $Q$. So, (6.3) and (6.4) apply to $J$.

Since $h \in \mathbb{k}[v, v w]$ and $\mathbb{k}$ is algebraically closed, we have

$$
h=\left(v w-a_{1} v^{2}\right) \cdots\left(v w-a_{n} v^{2}\right) v^{k}
$$


for some $n, k \in \mathbb{N}$ and $a_{1}, \ldots, a_{n} \in \mathbb{k}$. Applying Lemma 6.1 repeatedly, we obtain that $Q / h Q$ is a noetherian $B$-bimodule.

From the exact sequence

$$
0 \rightarrow h Q / h J \rightarrow Q / K \rightarrow Q / h Q \rightarrow 0,
$$

it suffices to prove that $h Q / h J$ is a noetherian $B$-bimodule. We make a geometric argument to do so.

Graded ideals of $T$ correspond to subschemes of the weighted projective plane $\mathbb{P}(1,1,2)$. Note that $\mu$ acts on $\mathbb{P}(1,1,2)$ by $\mu(a: b: c)=(a-b: b: c)$.

Let $Y_{n}$ be the subset of $\mathbb{P}(1,1,2)$ defined by the vanishing of the polynomials in $J_{n}$, considered now as a subset of $T$. By the definition of $h$, for $n \geq n_{1}$ the polynomials in $J_{n}$ have no nontrivial common factor, and so $\operatorname{dim} Y_{n} \leq 0$. By (6.3) and (6.4), we have

$$
Y_{n+1} \subseteq Y_{n} \cap \mu\left(Y_{n}\right)
$$

for $n \geq n_{1}$. It follows that there exists $n_{2} \geq n_{1}$ such that

$$
Y_{n+1}=Y_{n}=\mu\left(Y_{n}\right)
$$

for $n \geq n_{2}$. Let $Y:=Y_{n_{2}}$. Since $\mu$-orbits in $\mathbb{P}(1,1,2)$ are either infinite or trivial, each point of $Y$ is $\mu$-invariant. Note that $Y$ is the subset of $\mathbb{P}(1,1,2)$ defined by $J$, considered as an ideal of $T$.

Let $P$ be an associated prime of $J$. Since $J$ is graded, $P$ is graded. By using the Nullstellensatz, with the fact that $\operatorname{dim} Y \leq 0$, we get that either $P=T_{+}$, or $P$ defines some point $(a: b: c) \in Y$. In the first case, certainly $y \in P$. In the second case, $(a: b: c)=\mu(a: b: c)=(a-b: b: c)$ and so $b=0$. Again, $y \in P$.

The radical $\sqrt{J}$ is the intersection of the associated primes of $J$. Since $y$ is contained in all associated primes, $y \in \sqrt{J}$. Thus, there is some $n$ such that $y^{n}=v^{n} \in J$. So, $h Q / h J$ is a factor of $h Q / h v^{n} Q$. Applying Lemma 6.1 again, we see that $h Q / h J$ is a noetherian $B$-bimodule, as desired.

We now prove Proposition 0.12. In fact, we show:

Proposition 6.6. The ring $Q$ is noetherian as a B-bimodule. As a consequence, $B$ satisfies ACC on two-sided ideals.

Proof. Let $\mathbb{k}^{\prime}$ be an algebraic closure of $\mathbb{k}$. If $Q \otimes_{\mathbb{k}} \mathbb{k}^{\prime}$ were a noetherian bimodule over $B \otimes_{\mathbb{k}} \mathbb{K}^{\prime}$, then $Q$ would be a noetherian $B$-bimodule; this holds as $\mathbb{k}^{\prime}$ is faithfully flat over $\mathbb{k}$ [Goodearl and Warfield 2004, Exercise 17T]. So it suffices to prove the result in the case that $\mathbb{k}$ is algebraically closed. By standard arguments, it is sufficient to show that $Q$ satisfies ACC on graded $B$-subbimodules, or equivalently, that any nonzero graded $B$-subbimodule of $Q$ is finitely generated. 
Let $K$ be a nonzero graded $B$-subbimodule of $Q$. Since $B \supseteq Q p=p Q$ by Lemma 3.2(c), we have that $K=B K B \supseteq Q p K p Q$. Since $Q$ is noetherian, there is a finite-dimensional graded vector space $V \subseteq K$ with $Q p K p Q=Q p V p Q$.

By Proposition 6.2, the $B$-bimodule $Q / Q p V p Q$ is noetherian. Thus the $B$ subbimodule $K / Q p V p Q$ of $Q / Q p V p Q$ is finitely generated. So, there is a finitedimensional vector space $W \subseteq K$ such that $K=B W B+Q p V p Q \subseteq B W B+B V B$. As $V, W \subseteq K$, certainly $K \supseteq B W B+B V B$. Thus, $K$ is finitely generated by $V+W$, as needed.

\section{Appendix}

We first give a general result from ring theory to which we were not able to find a reference; it is the converse to [Rogalski 2014, Lemma 2.11]. We then finish by presenting Maple and Macaulay 2 routines and proofs of computational claims asserted above.

A result in ring theory. Consider the following setting. Let $T=\mathbb{k}\left\langle t_{1}, \ldots, t_{n}\right\rangle$ be the free algebra. Set $\operatorname{deg} t_{i}=d_{i} \in \mathbb{Z}_{\geq 1}$, and grade $T$ by the induced grading. Suppose that $\pi: T \rightarrow A$ is a surjective homomorphism of graded algebras, and let $a_{i}=\pi\left(t_{i}\right)$. By definition, the $a_{i}$ generate $A$ as an algebra. Let $J=\operatorname{ker} \pi$. Consider the map

$$
\alpha: A\left[-d_{1}\right] \oplus \cdots \oplus A\left[-d_{n}\right] \stackrel{\left(a_{1}, \ldots, a_{n}\right)}{\longrightarrow} A
$$

that sends $\left(r_{1}, \ldots, r_{n}\right) \mapsto \sum_{i=1}^{n} a_{i} r_{i}$. Note that $\alpha$ is a homomorphism of graded right $A$-modules, and set $K=\operatorname{ker} \alpha$. Let $b^{1}, \ldots, b^{m}$ be homogeneous elements of $K$, where $b^{j}=\left(b_{1}^{j}, \ldots, b_{n}^{j}\right) \in A\left[-d_{1}\right] \oplus \cdots \oplus A\left[-d_{n}\right]$. For all $1 \leq i \leq n$ and $1 \leq j \leq m$, choose homogeneous elements $\tilde{b}_{i}^{j} \in T$ so that $\pi\left(\tilde{b}_{i}^{j}\right)=b_{i}^{j}$. Let $q_{j}=\sum_{i=1}^{n} t_{i} \tilde{b}_{i}^{j}$. (Note that the $q_{i}$ are homogeneous; in fact, $\operatorname{deg} q_{j}=\operatorname{deg} b^{j}$.)

Proposition A.1. Retain the notation above. If $\left\{b^{1}, \ldots, b^{m}\right\}$ generate $K$ as a right $A$-module, then $\left\{q_{1}, \ldots, q_{m}\right\}$ generate $J$ as an ideal of $T$.

Proof. Let $J^{\prime}$ be the ideal of $T$ generated by $q_{1}, \ldots, q_{m}$. Since

$$
\pi\left(q_{j}\right)=\sum_{i} \pi\left(t_{i}\right) \pi\left(\tilde{b}_{i}^{j}\right)=\sum_{i} a_{i} b_{i}^{j}=\alpha\left(b^{j}\right)=0,
$$

we get that $J^{\prime} \subseteq J$.

We prove by induction that $J_{k}^{\prime}=J_{k}$ for all $k \in \mathbb{N}$. Certainly $J_{0}^{\prime}=J_{0}=0$. Assume that we have shown that $J_{<k}^{\prime}=J_{<k}$, and let $h \in J_{k}$. Because $T$ is generated by $t_{1}, \ldots, t_{n}$, there are homogeneous elements $f_{1}, \ldots, f_{n} \in T$, with $\operatorname{deg} f_{i}=k-d_{i}$, 
such that $h=\sum_{i} t_{i} f_{i}$. Then

$$
0=\pi(h)=\sum_{i=1}^{n} a_{i} \pi\left(f_{i}\right)=\alpha\left(\pi\left(f_{1}\right), \ldots, \pi\left(f_{n}\right)\right) .
$$

Since the $b^{j}$ generate $K=\operatorname{ker} \alpha$, there are homogeneous elements $r_{1}, \ldots, r_{m} \in A$ with $\left(\pi\left(f_{1}\right), \ldots, \pi\left(f_{n}\right)\right)=\sum_{j=1}^{m} b^{j} r_{j}$. Let $\tilde{r}_{1}, \ldots, \tilde{r}_{m}$ be homogeneous lifts of $r_{1}, \ldots, r_{m}$. Then for each $i$ we have

$$
\pi\left(f_{i}\right)=\sum_{j} b_{i}^{j} r_{j}=\sum_{j} \pi\left(\tilde{b}_{i}^{j} \tilde{r}_{j}\right)
$$

So, $f_{i}-\sum_{j} \tilde{b}_{i}^{j} \tilde{r}_{j} \in J=\operatorname{ker} \pi$. Since deg $f_{i}=k-d_{i}<k$, each $f_{i}-\sum_{j} \tilde{b}_{i}^{j} \tilde{r}_{j} \in J^{\prime}$. Thus $J^{\prime}$ contains

$$
\sum_{i} t_{i} f_{i}-\sum_{i} t_{i}\left(\sum_{j} \tilde{b}_{i}^{j} \tilde{r}_{j}\right)=h-\sum_{j}\left(\sum_{i} t_{i} \tilde{b}_{i}^{j}\right) \tilde{r}_{j}=h-\sum_{j} q_{j} \tilde{r}_{j} .
$$

As $\sum_{i} t_{i} \tilde{b}_{i}^{j}=q_{j} \in J^{\prime}$ by definition, we have $\sum_{j} q_{j} \tilde{r}_{j} \in J^{\prime}$. Therefore, $h \in J_{k}^{\prime}$.

Proof of assertions: Maple routines. We begin with the following Maple routine.

Routine A.2. A Maple routine to compute the kernel of $\lambda_{a}$ at a specific degree $n$ is presented as follows.

Recall from Lemma 1.1(b) that a $\mathbb{k}$-vector space basis of $U\left(W_{+}\right)_{n}$ is given by partitions of $n$. Moreover, we employ Lemma 1.3 and (1.4) to input a function $f(i, j)=\lambda_{a}\left(e_{i}\right)^{\mu^{j}}$, considered as an element of $\mathbb{k}[x, y]$.

with (combinat, partition): with(LinearAlgebra):

\# Choose value of $n$

$\mathrm{n}:=1$;

$N:=\operatorname{partition}(n): \quad f:=(i, j)->((x-j * y)-(i-1) * a * y) * y^{-}(i-1):$

Given a partition $d:=\left(n_{1}, \ldots, n_{k}\right)$ of $n$, we create a list of double-indexed entries $m=\left(m\left[i_{1}, j_{1}\right], \ldots, m\left[i_{k}, j_{k}\right]\right)$. Here, $i_{\ell}=n_{\ell}$, and $j_{1}=0$ with $j_{\ell}=j_{\ell-1}+n_{\ell-1}$ for $\ell \geq 2$. Then

$$
\lambda_{a}\left(e_{n_{1}} \cdots e_{n_{k}}\right)=m\left[i_{1}, j_{1}\right] \cdots m\left[i_{k}, j_{k}\right],
$$

denoted by $P$. (Here, $P$ is in list form, which we put in matrix form later for multiplication. The $k$-loop enables us to form the product of elements $m\left[i_{*}, j_{*}\right]$.) $\mathrm{P}:=[]$ :

for d from 1 to nops(N) do $\mathrm{M}:=[]:$ $j[1]:=0:$

for 1 from 1 to $\operatorname{nops}(\mathrm{N}[\mathrm{d}])$ do

$$
j[1+1]:=j[1]+N[d][1]: \quad M:=[o p(M), f(N[d][1], j[1])]: \quad S[0]:=1:
$$

for $k$ from 1 to nops(M) do $\mathrm{S}[\mathrm{k}]:=\mathrm{S}[\mathrm{k}-1] * \mathrm{M}[\mathrm{k}]:$

end do: end do:

$P:=[o p(P), \operatorname{expand}(S[\operatorname{nops}(M)])]$ :

end do: 
Next, we define an arbitrary element of $\lambda_{a}\left(U\left(W_{+}\right)_{n}\right)$, namely $p:=\sum_{i=1}^{k} b_{i} \lambda_{a}\left(e_{n_{i}}\right)$. $\mathrm{B}:=[]$;

for $i$ from 1 to nops(N) do $B:=[o p(B), b[i]]:$ end do:

Bvec: $=$ convert $(B$, Matrix $)$ : Pvec : =convert $(\mathrm{P}$, Matrix $)$ :

$\mathrm{q}:=$ Multiply (Bvec, Transpose (Pvec)) :

$\mathrm{p}:=\operatorname{expand}(\mathrm{q}[1][1])$ :

Then we set the coefficients of $p$ equal to 0 and solve for the $b_{i}$. We rule out the case when $a=0,1$.

Coeffs: $=[\operatorname{coeffs}(\operatorname{collect}(\mathrm{p},[\mathrm{x}, \mathrm{y}]$, 'distributed'),$[\mathrm{x}, \mathrm{y}])]$ : solve ( $[o p($ Coeffs) $, a<>0, a<>1])$;

Note that the number of free $b_{i}$ equals the $\mathbb{k}$-vector space dimension of $\left(\operatorname{ker} \lambda_{a}\right)_{n}$.

We continue by verifying the claim from the proof of Lemma 2.6.

Claim A.3. Retain the notation from Section 2, especially that in Lemma 2.6. We have that $J_{5} A(a)_{2} \nsubseteq J_{6} A(a)_{1}$.

Proof. Nonzero elements in $J_{5}$ arise as elements of $(u-a v) v A(a)_{3}$ that are divisible by $u$ on the left. We obtain that

$$
\begin{aligned}
& (u-a v) v A(a)_{3} \\
& \quad=\mathbb{k}\left[\left(u v-a v^{2}\right)\left(u^{3}\right)\right] \oplus \mathbb{k}\left[\left(u v-a v^{2}\right)(u(u-a v) v)\right] \oplus \mathbb{k}\left[\left(u v-a v^{2}\right)\left((u-2 a v) v^{2}\right)\right] \\
& \quad=\mathbb{k}\left[r_{1}\right] \oplus \mathbb{k}\left[r_{2}\right] \oplus \mathbb{k}\left[r_{3}\right],
\end{aligned}
$$

where

$$
\begin{aligned}
& r_{1}:=u^{4} v-(3+a) u^{3} v^{2}+(6+6 a) u^{2} v^{3}-(6+18 a) u v^{4}+24 a v^{5}, \\
& r_{2}:=u^{3} v^{2}-(2+2 a) u^{2} v^{3}+\left(2+5 a+a^{2}\right) u v^{4}-\left(6 a+2 a^{2}\right) v^{5}, \\
& r_{3}:=u^{2} v^{3}-(1+3 a) u v^{4}+\left(2 a+2 a^{2}\right) v^{5} .
\end{aligned}
$$

We see this as $v^{k} u=u v^{k}-k v^{k+1}$ for all $k \geq 1, v u^{2}=u^{2} v-2 u v^{2}+2 v^{3}$, $v^{2} u^{2}=u^{2} v^{2}-4 u v^{3}+6 v^{4}, v u^{3}=u^{3} v-3 u^{2} v^{2}+6 u v^{3}-6 v^{4}$, and $v^{2} u^{3}=$ $u^{3} v^{2}-6 u^{2} v^{3}+18 u v^{4}-24 v^{5}$ in $R$. Eliminating the $v^{5}$ term of $r_{1}, r_{2}, r_{3}$, we get that $J_{5}$ is generated by

$$
\begin{aligned}
& s_{1}:=(3+a) r_{1}+12 r_{2}, \\
& s_{2}:=(1+a) r_{1}-12 r_{3}, \\
& s_{3}:=(1+a) r_{2}+(3+a) r_{3} .
\end{aligned}
$$

By way of contradiction, suppose that $J_{5} A(a)_{2} \subseteq J_{6} A(a)_{1}$. Recall that $J \subseteq L$, where $L:=u R \cap(u-a v) v R$. Further, $J_{6}=L_{6}$, and $L=r R$ for

$$
r=u\left(u v+(1-a) v^{2}\right)=\left(u v-a v^{2}\right)(u+2 v) .
$$

So, $s_{i}=r\left(c_{i 1} u^{2}+c_{i 2} u v+c_{i 3} v^{2}\right) \in J_{5} \subseteq r R_{2}$, for some $c_{i j} \in \mathbb{k}$. We produce these coefficients $c_{i j}$ below. 


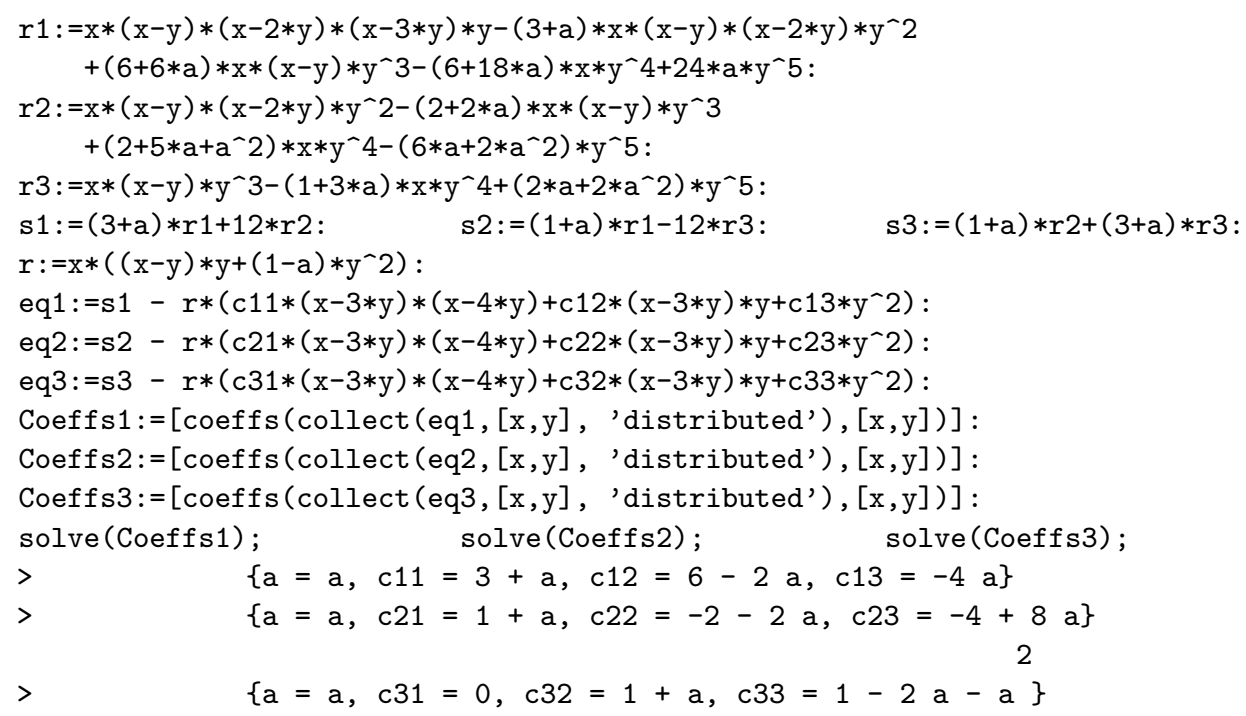

Therefore,

$$
\begin{aligned}
& s_{1}=r\left((3+a) u^{2}+(6-2 a) u v-4 a v^{2}\right), \\
& s_{2}=r\left((1+a) u^{2}-(2+2 a) u v-(4-8 a) v^{2}\right), \\
& s_{3}=r\left((1+a) u v+\left(1-2 a-a^{2}\right) v^{2}\right) .
\end{aligned}
$$

By assumption, for $i=1,2,3$ we have $s_{i}(u-a v) v=w_{i} u$ for some $w_{i} \in J_{6}$. Take an arbitrary element of $J_{6}=L_{6}=r R_{3}$, namely $r\left(d_{i 1} u^{3}+d_{i 2} u^{2} v+d_{i 3} u v^{2}+d_{i 4} v^{3}\right)$ for $d_{i j} \in \mathbb{k}$. Then, for some $\alpha_{i} \in \mathbb{k}$,

$$
p_{i}:=s_{i}(u-a v) v=\alpha_{i} r\left(d_{i 1} u^{4}+d_{i 2} u^{2} v u+d_{i 3} u v^{2} u+d_{i 4} v^{3} u\right) .
$$

Continuing with the code we enter:

$$
\begin{aligned}
& \mathrm{s} 1:=\mathrm{r} *\left((3+\mathrm{a}) *(\mathrm{x}-3 * \mathrm{y}) *(\mathrm{x}-4 * \mathrm{y})+(6-2 * \mathrm{a}) *(\mathrm{x}-3 * \mathrm{y}) * \mathrm{y}-4 * \mathrm{a} * \mathrm{y}^{\wedge} 2\right): \\
& \mathrm{s} 2:=\mathrm{r} *\left((1+\mathrm{a}) *(\mathrm{x}-3 * \mathrm{y}) *(\mathrm{x}-4 * \mathrm{y})-(2+2 * \mathrm{a}) *(\mathrm{x}-3 * \mathrm{y}) * \mathrm{y}-(4-8 * \mathrm{a}) * \mathrm{y}^{\wedge} 2\right): \\
& \mathrm{s} 3:=\mathrm{r} *\left((1+\mathrm{a}) *(\mathrm{x}-3 * \mathrm{y}) * \mathrm{y}+\left(1-2 * \mathrm{a}-\mathrm{a}^{-2}\right) * \mathrm{y}-2\right): \\
& \mathrm{p} 1:=\mathrm{s} 1 *(\mathrm{x}-(5+\mathrm{a}) * \mathrm{y}) * \mathrm{y}: \quad \mathrm{p} 2:=\mathrm{s} 2 *(\mathrm{x}-(5+\mathrm{a}) * \mathrm{y}) * \mathrm{y}: \quad \mathrm{p} 3:=\mathrm{s} 3 *(\mathrm{x}-(5+\mathrm{a}) * \mathrm{y}) * \mathrm{y}: \\
& \text { Eq1:=p1 }-\mathrm{alpha} 1 * \mathrm{r} *(\mathrm{~d} 11 *(\mathrm{x}-3 * \mathrm{y}) *(\mathrm{x}-4 * \mathrm{y}) *(\mathrm{x}-5 * \mathrm{y}) *(\mathrm{x}-6 * \mathrm{y})
\end{aligned}
$$$$
+\mathrm{d} 12 *(\mathrm{x}-3 * \mathrm{y}) *(\mathrm{x}-4 * \mathrm{y}) * \mathrm{y} *(\mathrm{x}-6 * \mathrm{y})
$$$$
\left.+\mathrm{d} 13 *(\mathrm{x}-3 * \mathrm{y}) * \mathrm{y}^{\wedge} 2 *(\mathrm{x}-6 * \mathrm{y})+\mathrm{d} 14 * \mathrm{y}^{\wedge} 3 *(\mathrm{x}-6 * \mathrm{y})\right):
$$

Eq2: $=$ p2 - alpha2 $* r *(\mathrm{~d} 21 *(\mathrm{x}-3 * \mathrm{y}) *(\mathrm{x}-4 * \mathrm{y}) *(\mathrm{x}-5 * \mathrm{y}) *(\mathrm{x}-6 * \mathrm{y})$

$$
\begin{aligned}
& +\mathrm{d} 22 *(\mathrm{x}-3 * \mathrm{y}) *(\mathrm{x}-4 * \mathrm{y}) * \mathrm{y} *(\mathrm{x}-6 * \mathrm{y}) \\
& \left.+\mathrm{d} 23 *(\mathrm{x}-3 * \mathrm{y}) * \mathrm{y}^{\wedge} 2 *(\mathrm{x}-6 * \mathrm{y})+\mathrm{d} 24 * \mathrm{y}^{\wedge} 3 *(\mathrm{x}-6 * \mathrm{y})\right):
\end{aligned}
$$

Eq3: $=$ p3 - alpha3*r* $(\mathrm{d} 31 *(\mathrm{x}-3 * \mathrm{y}) *(\mathrm{x}-4 * \mathrm{y}) *(\mathrm{x}-5 * \mathrm{y}) *(\mathrm{x}-6 * \mathrm{y})$

$$
\begin{aligned}
& +\mathrm{d} 32 *(\mathrm{x}-3 * \mathrm{y}) *(\mathrm{x}-4 * \mathrm{y}) * \mathrm{y} *(\mathrm{x}-6 * \mathrm{y}) \\
& \left.+\mathrm{d} 33 *(\mathrm{x}-3 * \mathrm{y}) * \mathrm{y}^{\wedge} 2 *(\mathrm{x}-6 * \mathrm{y})+\mathrm{d} 34 * \mathrm{y}^{\wedge} 3 *(\mathrm{x}-6 * \mathrm{y})\right):
\end{aligned}
$$

CCoeffs $1:=[\operatorname{coeffs}(\operatorname{collect}(\mathrm{Eq} 1,[\mathrm{x}, \mathrm{y}]$, 'distributed'),$[\mathrm{x}, \mathrm{y}])]$ : CCoeffs $2:=[\operatorname{coeffs}(\operatorname{collect}(E q 2,[\mathrm{x}, \mathrm{y}]$, 'distributed'), $[\mathrm{x}, \mathrm{y}])]$ : CCoeffs $3:=[\operatorname{coeffs}(\operatorname{collect}($ Eq3,$[\mathrm{x}, \mathrm{y}]$, 'distributed') $[\mathrm{x}, \mathrm{y}])]$ :
L1: =solve (CCoeffs1):
L2: =solve (CCoeffs2) :
L3:=solve (CCoeffs3) : 
for $i$ from 1 to nops([L1]) do $\operatorname{print}(\mathrm{L} 1[\mathrm{i}][1])$;

end do;

$>\quad a=9, \quad a=1$

for $i$ from 1 to nops([L2]) do

$>\quad a=1, \quad a=1 / 2$

for $i$ from 1 to nops([L3]) do print(L3[i][1]);

end do;

end do;

$a=1, \quad a=\operatorname{Root0f}(-2-3 \ldots+Z Z)-1$

So in order for (A.4) to hold for $i=1,2,3$, we must have $a=1$. This yields a contradiction, as desired.

We now verify the claim from the proof of Proposition 2.8.

Claim A.5. Retain the notation from Section 2, especially that in Proposition 2.8. We have that $h_{2}, h_{3}, e_{1} h_{1}, h_{1} e_{1}$ are $\mathbb{k}$-linearly independent and that

$$
\begin{aligned}
& h_{4}=2 a(2 a+1) h_{2}-h_{3}-(6+4 a) e_{1} h_{1}+(2+4 a) h_{1} e_{1}, \\
& h_{5}=4 a^{2} h_{2}-h_{3}-(4+4 a) e_{1} h_{1}+(4 a) h_{1} e_{1} .
\end{aligned}
$$

Proof. This is established simply by considering the linear combination

$$
c_{1} h_{2}+c_{2} h_{3}+c_{3} h_{4}+c_{4} h_{5}+c_{5} e_{1} h_{1}+c_{6} h_{1} e_{1},
$$

setting the coefficients of the basis elements of $U\left(W_{+}\right)_{6}$ equal to 0 , and solving for $c_{1}, \ldots, c_{6}$. By Lemma 1.1(a), the basis elements of $U\left(W_{+}\right)_{6}$ are

$$
e_{1}^{6}, e_{1}^{4} e_{2}, e_{1}^{2} e_{2}^{2}, e_{2}^{3}, e_{1}^{3} e_{3}, e_{1} e_{2} e_{3}, e_{3}^{2}, e_{1}^{2} e_{4}, e_{2} e_{4}, e_{1} e_{5}, e_{6} .
$$

So, we establish the claim via the following Maple routine:

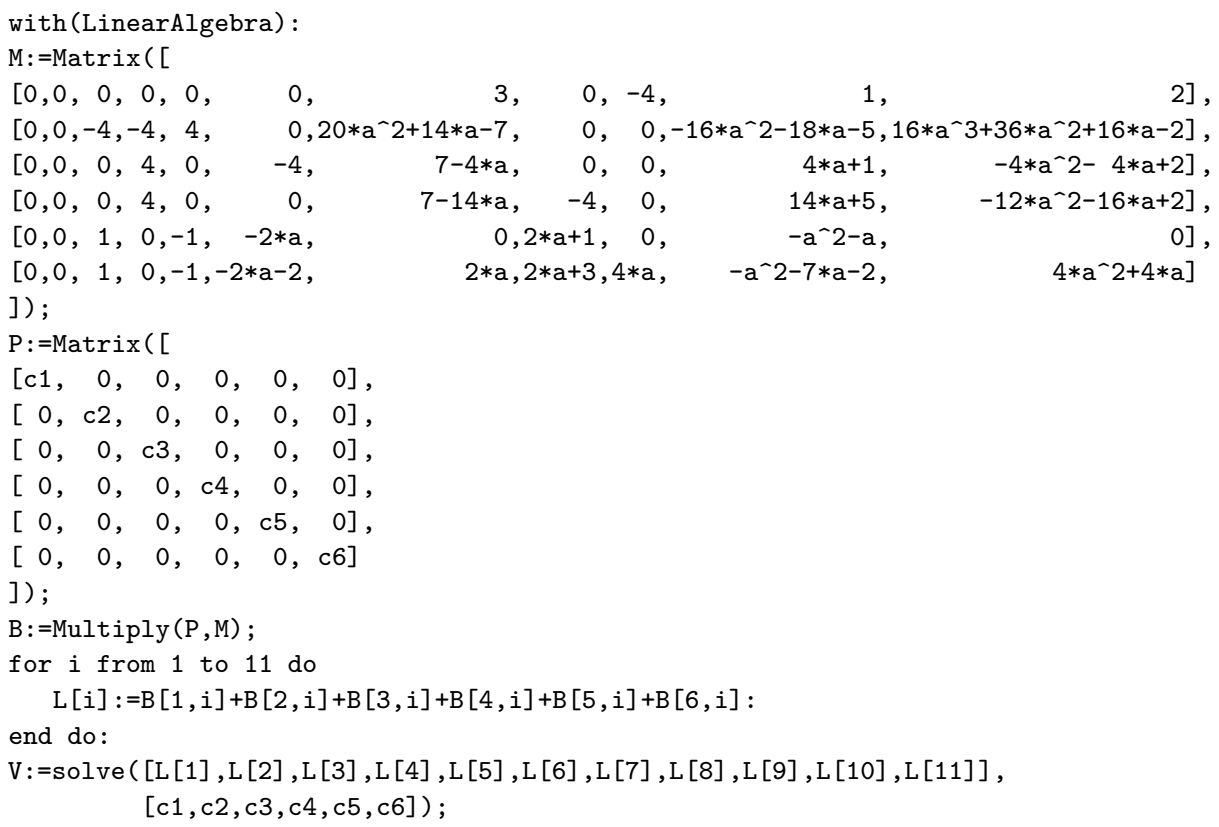


$>[[c 1=-2(c 3+2 c 3 a+2 c 4 a) a, \quad c 2=c 3+c 4, \quad c 3=c 3, \quad c 4=c 4$,

$c 5=6 c 3+4 c 4+4 c 3 a+4 c 4 a, c 6=-2 c 3-4 c 3 a-4 c 4 a]]$

eval (V, $[\mathrm{c} 3=1, \mathrm{c} 4=0]$ );

$>[[c 1=-2(2 a+1) a, c 2=1,1=1,0=0, c 5=6+4 a, c 6=-2-4 a]]$

$\operatorname{eval}(\mathrm{V},[\mathrm{c} 3=0, \mathrm{c} 4=1])$;

$>$

2

$$
[\mathrm{c} 1=-4 \mathrm{a}, \mathrm{c} 2=1,0=0,1=1, \mathrm{c} 5=4+4 \mathrm{a}, \mathrm{c} 6=-4 \mathrm{a}]]
$$

We now verify the claims from the proof of Lemma 5.12.

Claim A.6. Retain the notation from Lemma 5.12.

(a) $b_{5} Q+b_{6} Q+b_{7} Q \ni x(x y-y z)\left(x y z+y^{2} z\right)=(u v-v w)(u+2 v)(u+4 v) v w$.

(b) $\left(M^{\prime} \cap \operatorname{ker} \eta\right) \supseteq h Q_{i}$ for $i \leq 2$, where

$$
h=(u v-v w)(u+2 v)\left(v^{3} w-v^{2} w^{2}\right)=(x y-y z) x\left(y^{3} z-y^{2} z^{2}\right) .
$$

Proof. (a) Using Lemma 1.3 and (1.4), we see that $-\frac{1}{6} b_{5} u+b_{5} v+\frac{1}{6} b_{6}=$ $(u v-v w)(u+2 v)(u+4 v) v w$ :

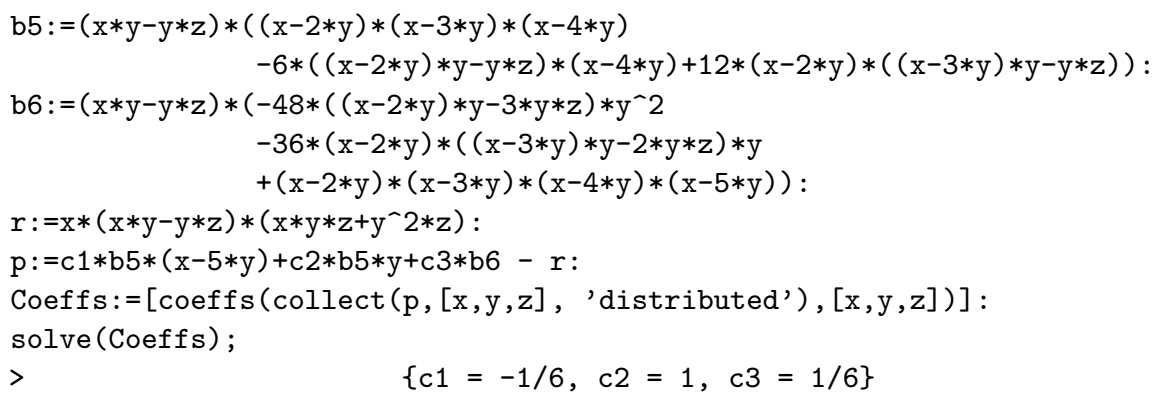

(b) It is easy to see that $\eta(h)=0$, so it suffices to show that $h Q_{0}, h Q_{1}, h Q_{2}$ are in $M^{\prime}:=b_{5} B+b_{6} B+b_{7} B$. Recall that $Q$ is the subalgebra of $S$ generated by $u, v, v w$, and $B$ is the subalgebra of $S$ generated by $u, u v-v w$. Since $\operatorname{deg}(h)=7$,

$$
\begin{aligned}
& h Q_{0}=\left\{c_{1} h \mid c_{1} \in \mathbb{k}\right\} \\
& h Q_{1}=\left\{c_{2} h u+c_{3} h v \mid c_{i} \in \mathbb{k}\right\} \\
& h Q_{2}=\left\{c_{4} h u^{2}+c_{5} h u v+c_{6} h v^{2}+c_{7} h v w \mid c_{i} \in \mathbb{k}\right\}
\end{aligned}
$$

and moreover,

$$
\begin{aligned}
& M_{7}^{\prime}=\left\{d_{1} b_{5} u^{2}+d_{2} b_{5}(u v-v w)+d_{3} b_{6} u+d_{4} b_{7} \mid d_{i} \in \mathbb{R}\right\}, \\
& M_{8}^{\prime}=\left\{d_{5} b_{5} u^{3}+d_{6} b_{5} u(u v-v w)+d_{7} b_{5}(u v-v w) u\right. \\
& \left.\quad+d_{8} b_{6} u^{2}+d_{9} b_{6}(u v-v w)+d_{10} b_{7} u \mid d_{i} \in \mathbb{k}\right\}, \\
& \begin{array}{r}
M_{9}^{\prime}=\left\{d_{11} b_{5} u^{4}+d_{12} b_{5} u^{2}(u v-v w)+d_{13} b_{5} u(u v-v w) u+d_{14} b_{5}(u v-v w) u^{2}\right. \\
+d_{15} b_{5}(u v-v w)^{2}+d_{16} b_{6} u^{3}+d_{17} b_{6} u(u v-v w) \\
\left.+d_{18} b_{6}(u v-v w) u+d_{19} b_{7} u^{2}+d_{20} b_{7}(u v-v w) \mid d_{i} \in \mathbb{k}\right\},
\end{array}
\end{aligned}
$$


Continuing with the code in part (a), we enter:

$$
\begin{aligned}
\mathrm{b} 7:=(\mathrm{x} * \mathrm{y}-\mathrm{y} * \mathrm{z}) * & ((\mathrm{x}-2 * \mathrm{y}) *(\mathrm{x}-3 * \mathrm{y}) *(\mathrm{x}-4 * \mathrm{y}) *(\mathrm{x}-5 * \mathrm{y}) *(\mathrm{x}-6 * \mathrm{y}) \\
& -40 *(((\mathrm{x}-2 * \mathrm{y}) * \mathrm{y}-\mathrm{y} * \mathrm{z}) *((\mathrm{x}-4 * \mathrm{y}) * \mathrm{y}-\mathrm{y} * \mathrm{z}) *(\mathrm{x}-6 * \mathrm{y}) \\
& -3 *((\mathrm{x}-2 * \mathrm{y}) * \mathrm{y}-\mathrm{y} * \mathrm{z}) *(\mathrm{x}-4 * \mathrm{y}) *((\mathrm{x}-5 * \mathrm{y}) * \mathrm{y}-\mathrm{y} * \mathrm{z}) \\
& +3 *(\mathrm{x}-2 * \mathrm{y}) *((\mathrm{x}-3 * \mathrm{y}) * \mathrm{y}-\mathrm{y} * \mathrm{z}) *((\mathrm{x}-5 * \mathrm{y}) * \mathrm{y}-\mathrm{y} * \mathrm{z}))):
\end{aligned}
$$

$\mathrm{h}:=(\mathrm{x} * \mathrm{y}-\mathrm{y} * \mathrm{z}) * \mathrm{x} *\left(\mathrm{y}^{\wedge} 3 * \mathrm{z}-\mathrm{y}^{\wedge} 2 * \mathrm{z}^{\wedge} 2\right):$

$\mathrm{hQ0}:=\mathrm{c} 1 * \mathrm{~h}:$

$\mathrm{hQ1}:=\mathrm{c} 2 * \mathrm{~h} *(\mathrm{x}-7 * \mathrm{y})+\mathrm{c} 3 * \mathrm{~h} * \mathrm{y}:$

$\mathrm{hQ2}:=\mathrm{c} 4 * \mathrm{~h} *(\mathrm{x}-7 * \mathrm{y}) *(\mathrm{x}-8 * \mathrm{y})+\mathrm{c} 5 * \mathrm{~h} *(\mathrm{x}-7 * \mathrm{y}) * \mathrm{y}+\mathrm{c} 6 * \mathrm{~h} * \mathrm{y}-2+\mathrm{c} 7 * \mathrm{~h} * \mathrm{y} * \mathrm{z}:$

$\mathrm{m} 7:=\mathrm{d} 1 * \mathrm{~b} 5 *(\mathrm{x}-5 * \mathrm{y}) *(\mathrm{x}-6 * \mathrm{y})+\mathrm{d} 2 * \mathrm{~b} 5 *((\mathrm{x}-5 * \mathrm{y}) * \mathrm{y}-\mathrm{y} * \mathrm{z})+\mathrm{d} 3 * \mathrm{~b} 6 *(\mathrm{x}-6 * \mathrm{y})+\mathrm{d} 4 * \mathrm{~b} 7:$

$\mathrm{m} 8:=\mathrm{d} 5 * \mathrm{~b} 5 *(\mathrm{x}-5 * \mathrm{y}) *(\mathrm{x}-6 * \mathrm{y}) *(\mathrm{x}-7 * \mathrm{y})+\mathrm{d} 6 * \mathrm{~b} 5 *(\mathrm{x}-5 * \mathrm{y}) *((\mathrm{x}-6 * \mathrm{y}) * \mathrm{y}-\mathrm{y} * \mathrm{z})$

$+\mathrm{d} 7 * \mathrm{~b} 5 *((\mathrm{x}-5 * \mathrm{y}) * \mathrm{y}-\mathrm{y} * \mathrm{z}) *(\mathrm{x}-7 * \mathrm{y})+\mathrm{d} 8 * \mathrm{~b} 6 *(\mathrm{x}-6 * \mathrm{y}) *(\mathrm{x}-7 * \mathrm{y})$

$+\mathrm{d} 9 * \mathrm{~b} 6 *((\mathrm{x}-6 * \mathrm{y}) * \mathrm{y}-\mathrm{y} * \mathrm{z})+\mathrm{d} 10 * \mathrm{~b} 7 *(\mathrm{x}-7 * \mathrm{y}):$

$\mathrm{m} 9:=\mathrm{d} 11 * \mathrm{~b} 5 *(\mathrm{x}-5 * \mathrm{y}) *(\mathrm{x}-6 * \mathrm{y}) *(\mathrm{x}-7 * \mathrm{y}) *(\mathrm{x}-8 * \mathrm{y})$

$+\mathrm{d} 12 * \mathrm{~b} 5 *(\mathrm{x}-5 * \mathrm{y}) *(\mathrm{x}-6 * \mathrm{y}) *((\mathrm{x}-7 * \mathrm{y}) * \mathrm{y}-\mathrm{y} * \mathrm{z})$

$+\mathrm{d} 13 * \mathrm{~b} 5 *(\mathrm{x}-5 * \mathrm{y}) *((\mathrm{x}-6 * \mathrm{y}) * \mathrm{y}-\mathrm{y} * \mathrm{z}) *(\mathrm{x}-8 * \mathrm{y})$

$+\mathrm{d} 14 * \mathrm{~b} 5 *((\mathrm{x}-5 * \mathrm{y}) * \mathrm{y}-\mathrm{y} * \mathrm{z}) *(\mathrm{x}-7 * \mathrm{y}) *(\mathrm{x}-8 * \mathrm{y})$

$+\mathrm{d} 15 * \mathrm{~b} 5 *((\mathrm{x}-5 * \mathrm{y}) * \mathrm{y}-\mathrm{y} * \mathrm{z}) *((\mathrm{x}-7 * \mathrm{y}) * \mathrm{y}-\mathrm{y} * \mathrm{z})+\mathrm{d} 16 * \mathrm{~b} 6 *(\mathrm{x}-6 * \mathrm{y}) *(\mathrm{x}-7 * \mathrm{y}) *(\mathrm{x}-8 * \mathrm{y})$

$+\mathrm{d} 17 * \mathrm{~b} 6 *(\mathrm{x}-6 * \mathrm{y}) *((\mathrm{x}-7 * \mathrm{y}) * \mathrm{y}-\mathrm{y} * \mathrm{z})+\mathrm{d} 18 * \mathrm{~b} 6 *((\mathrm{x}-6 * \mathrm{y}) * \mathrm{y}-\mathrm{y} * \mathrm{z}) *(\mathrm{x}-8 * \mathrm{y})$

$+\mathrm{d} 19 * \mathrm{~b} 7 *(\mathrm{x}-7 * \mathrm{y}) *(\mathrm{x}-8 * \mathrm{y})+\mathrm{d} 20 * \mathrm{~b} 7 *((\mathrm{x}-7 * \mathrm{y}) * \mathrm{y}-\mathrm{y} * \mathrm{z}):$

p7:=m7 - hQ0: p8:=m8 - hQ1: p9:=m9 - hQ2:

Coeffs $7:=[\operatorname{coeffs}(\operatorname{collect}(\mathrm{p} 7,[\mathrm{x}, \mathrm{y}, \mathrm{z}]$, 'distributed'), $\mathrm{x}, \mathrm{y}, \mathrm{z}])]$ :

Coeffs $8:=[\operatorname{coeffs}(\operatorname{collect}(\mathrm{p} 8,[\mathrm{x}, \mathrm{y}, \mathrm{z}]$, 'distributed'), $[\mathrm{x}, \mathrm{y}, \mathrm{z}])]$ :

Coeffs9: $=\left[\operatorname{coeffs}\left(\operatorname{collect}\left(\mathrm{p} 9,[\mathrm{x}, \mathrm{y}, \mathrm{z}],{ }^{\prime}\right.\right.\right.$ 'distributed'), $\left.\left.\left.\mathrm{x}, \mathrm{y}, \mathrm{z}\right]\right)\right]$ :

solve(Coeffs $7,[\mathrm{~d} 1, \mathrm{~d} 2, \mathrm{~d} 3, \mathrm{~d} 4]$ );

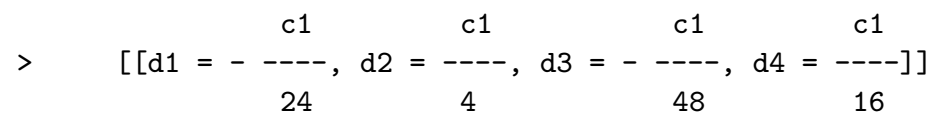

solve (Coeffs8, [d5, d6,d7, d8, d9, d10]);

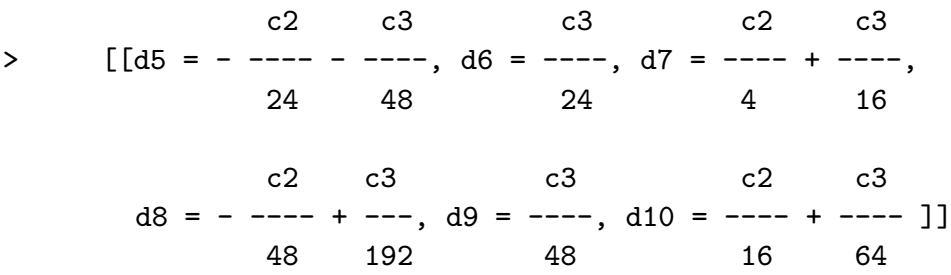

solve (Coeffs9, [d11, d12, d13, d14,d15,d16,d17, d18,d19,d20]);

$>\quad\left[\mathrm{d} 11=8 \mathrm{~d} 16+\begin{array}{ccccc}c 4 & c 6 & c 5 & c 7 \\ 8 & 144 & 18 & 18 & \end{array}\right.$

$$
\mathrm{d} 20=-108 \mathrm{~d} 16 \begin{array}{cccc}
9 \mathrm{c} 4 & \mathrm{c6} & 25 \mathrm{c5} & 11 \mathrm{c7} \\
---- & ---- & +--- & +----]]
\end{array}
$$

Thus, all arbitrary elements of $h Q_{0}, h Q_{1}, h Q_{2}$ are contained, respectively, in $M_{7}^{\prime}$, $M_{8}^{\prime}, M_{9}^{\prime}$, as desired. 
Proof of assertions: Macaulay2 routines. The following Macaulay 2 code verifies Lemma 4.2(b) and (4.6); see lines o7-o10 and line o13, respectively.

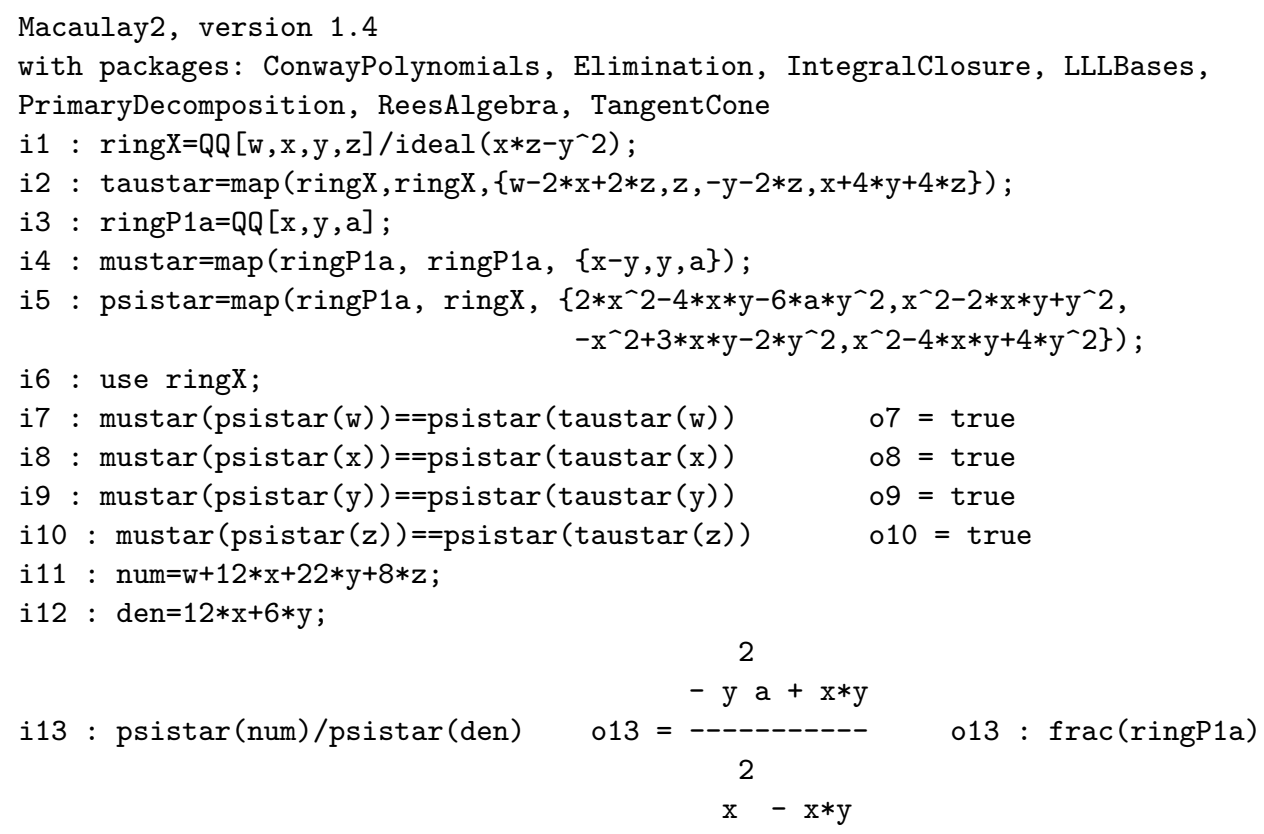

\section{Acknowledgements}

C. Walton was supported by the US National Science Foundation grant \#1550306. We thank MIT and the NSF for supporting a visit by the first author to MIT in February 2015, in which much of the work on this paper was done. We thank Jacques Alev, Jason Bell, and Lance Small for helpful discussions. In addition, we thank the referee for pointing us to [Conley and Martin 2007] and for prompting Remarks 3.14 and 5.15.

\section{References}

[Artin and Stafford 1995] M. Artin and J. T. Stafford, "Noncommutative graded domains with quadratic growth”, Invent. Math. 122:2 (1995), 231-276. MR 1358976 Zbl 0849.16022

[Conley and Martin 2007] C. H. Conley and C. Martin, "Annihilators of tensor density modules", J. Algebra 312:1 (2007), 495-526. MR 2320470 Zbl 1171.17008

[Goodearl and Warfield 2004] K. R. Goodearl and R. B. Warfield, Jr., An introduction to noncommutative Noetherian rings, 2nd ed., London Mathematical Society Student Texts 61, Cambridge University Press, 2004. MR 2080008 Zbl 1101.16001

[Harris 1992] J. Harris, Algebraic geometry: a first course, Graduate Texts in Mathematics 133, Springer, New York, 1992. MR 1182558 Zbl 0779.14001

[Hartshorne 1977] R. Hartshorne, Algebraic geometry, Graduate Texts in Mathematics 52, Springer, New York, 1977. MR 0463157 Zbl 0367.14001 
[Rogalski 2014] D. Rogalski, "An introduction to noncommutative projective algebraic geometry", lecture notes, 2014. arXiv 1403.3065

[Sierra 2011] S. J. Sierra, "Classifying birationally commutative projective surfaces", Proc. Lond. Math. Soc. (3) 103:1 (2011), 139-196. MR 2812504 Zbl 1226.14004

[Sierra and Walton 2014] S. J. Sierra and C. Walton, "The universal enveloping algebra of the Witt algebra is not noetherian”, Adv. Math. 262 (2014), 239-260. MR 3228429 Zbl 1297.14006

[Stafford 1985] J. T. Stafford, "On the ideals of a Noetherian ring", Trans. Amer. Math. Soc. 289:1 (1985), 381-392. MR 779071 Zbl 0566.16006

[Stafford and Zhang 1994] J. T. Stafford and J. J. Zhang, "Examples in non-commutative projective geometry", Math. Proc. Cambridge Philos. Soc. 116:3 (1994), 415-433. MR 1291750 Zbl 0821.16026

[Zhang 1996] J. J. Zhang, "Twisted graded algebras and equivalences of graded categories", Proc. London Math. Soc. (3) 72:2 (1996), 281-311. MR 1367080 Zbl 0852.16005

Received September 2, 2015. Revised May 4, 2016.

SUSAN J. SIERRA

SCHOOL OF MATHEMATICS

UNIVERSITY OF EDINBURGH

James Clerk Maxwell Building, Kings Buildings

MAYFIELD ROAD

EDINBURGH EH9 3JZ

UNITED KINGDOM

s.sierra@ed.ac.uk

Chelsea Walton

DEPARTMENT OF MATHEMATICS

TEMPLE UNIVERSITY

PhILADELPHIA, PA 19122

United STATES

notlaw@temple.edu 


\title{
PACIFIC JOURNAL OF MATHEMATICS
}

Founded in 1951 by E. F. Beckenbach (1906-1982) and F. Wolf (1904-1989)

$$
\text { msp.org/pjm }
$$

\section{EDITORS}

\author{
Don Blasius (Managing Editor) \\ Department of Mathematics \\ University of California \\ Los Angeles, CA 90095-1555 \\ blasius@math.ucla.edu
}

\author{
Paul Balmer \\ Department of Mathematics \\ University of California \\ Los Angeles, CA 90095-1555 \\ balmer@math.ucla.edu \\ Robert Finn \\ Department of Mathematics \\ Stanford University \\ Stanford, CA 94305-2125 \\ finn@math.stanford.edu \\ Sorin Popa \\ Department of Mathematics \\ University of California \\ Los Angeles, CA 90095-1555 \\ popa@math.ucla.edu
}

\author{
Vyjayanthi Chari \\ Department of Mathematics \\ University of California \\ Riverside, CA 92521-0135 \\ chari@math.ucr.edu \\ Kefeng Liu \\ Department of Mathematics \\ University of California \\ Los Angeles, CA 90095-1555 \\ liu@math.ucla.edu \\ Igor Pak \\ Department of Mathematics \\ University of California \\ Los Angeles, CA 90095-1555 \\ pak.pjm@gmail.com \\ Paul Yang \\ Department of Mathematics \\ Princeton University \\ Princeton NJ 08544-1000 \\ yang@math.princeton.edu
}

\section{PRODUCTION}

Silvio Levy, Scientific Editor, production@msp.org

\section{SUPPORTING INSTITUTIONS}

ACADEMIA SINICA, TAIPEI

CALIFORNIA INST. OF TECHNOLOGY

STANFORD UNIVERSITY

UNIV. OF BRITISH COLUMBIA

UNIV. OF CALIFORNIA, BERKELEY

UNIV. OF CALIFORNIA, DAVIS

UNIV. OF CALIFORNIA, LOS ANGELES

UNIV. OF CALIFORNIA, RIVERSIDE

UNIV. OF CALIFORNIA, SAN DIEGO

UNIV. OF CALIF., SANTA BARBARA
KEIO UNIVERSITY

MATH. SCIENCES RESEARCH INSTITUTE

NEW MEXICO STATE UNIV.

OREGON STATE UNIV.
Daryl Cooper

Department of Mathematics

University of California

Santa Barbara, CA 93106-3080 cooper@math.ucsb.edu

Jiang-Hua Lu

Department of Mathematics

The University of Hong Kong

Pokfulam Rd., Hong Kong

jhlu@maths.hku.hk

$$
\text { Jie Qing }
$$

Department of Mathematics

University of California

Santa Cruz, CA 95064

qing@ cats.ucsc.edu

\author{
UNIV. OF CALIF., SANTA CRUZ \\ UNIV. OF MONTANA \\ UNIV. OF OREGON \\ UNIV. OF SOUTHERN CALIFORNIA \\ UNIV. OF UTAH \\ UNIV. OF WASHINGTON \\ WASHINGTON STATE UNIVERSITY
}

These supporting institutions contribute to the cost of publication of this Journal, but they are not owners or publishers and have no responsibility for its contents or policies.

See inside back cover or msp.org/pjm for submission instructions.

The subscription price for 2016 is US $\$ 440 /$ year for the electronic version, and \$600/year for print and electronic.

Subscriptions, requests for back issues and changes of subscriber address should be sent to Pacific Journal of Mathematics, P.O. Box 4163, Berkeley, CA 94704-0163, U.S.A. The Pacific Journal of Mathematics is indexed by Mathematical Reviews, Zentralblatt MATH, PASCAL CNRS Index, Referativnyi Zhurnal, Current Mathematical Publications and Web of Knowledge (Science Citation Index).

The Pacific Journal of Mathematics (ISSN 0030-8730) at the University of California, c/o Department of Mathematics, 798 Evans Hall \#3840, Berkeley, CA 94720-3840, is published twelve times a year. Periodical rate postage paid at Berkeley, CA 94704, and additional mailing offices. POSTMASTER: send address changes to Pacific Journal of Mathematics, P.O. Box 4163, Berkeley, CA 94704-0163.

PJM peer review and production are managed by EditFLOW ${ }^{\circledR}$ from Mathematical Sciences Publishers.

PUBLISHED BY

\section{I. mathematical sciences publishers}

nonprofit scientific publishing

http://msp.org/

(C) 2016 Mathematical Sciences Publishers 


\section{PACIFIC JOURNAL OF MATHEMATICS}

Volume $284 \quad$ No. $2 \quad$ October 2016

Spherical CR Dehn surgeries

Miguel ACOsta

Degenerate flag varieties and Schubert varieties: a characteristic free approach

283

Giovanni Cerulli Irelli, Martina LANini and Peter

LITTELMANN

Solitons for the inverse mean curvature flow

Gregory Drugan, HoJoo LEE and GLEN WHEELER

Bergman theory of certain generalized Hartogs triangles

\section{LUKE D. EDHOLM}

Transference of certain maximal Hilbert transforms on the torus

DASHAN FAN, HUOXIONG WU and FAYOU ZHAO

The Turaev and Thurston norms

STEFAN FriedL, DANIEL S. Silver and Susan G. WiLLiams

A note on nonunital absorbing extensions

JAMES GABE

On nonradial singular solutions of supercritical biharmonic equations

Zongming GuO, JUNCHENG WeI and Wen YANG

Natural commuting of vanishing cycles and the Verdier dual

\section{DAVID B. MASSEY}

The nef cones of and minimal-degree curves in the Hilbert schemes of points on certain surfaces

ZHENBo QIN and YUPING TU

Smooth approximation of conic Kähler metric with lower Ricci curvature bound

\section{LIANGMING SHEN}

Maps from the enveloping algebra of the positive Witt algebra to regular algebras

Susan J. SierRa and Chelsea Walton 